Submitted to ApJ

Preprint typeset using $\mathrm{LT}_{\mathrm{E}} \mathrm{X}$ style emulateapj v. 5/2/11

\title{
EARLY-TYPE GALAXY ARCHEOLOGY: AGES, ABUNDANCE RATIOS, AND EFFECTIVE TEMPERATURES FROM
} FULL-SPECTRUM FITTING

\author{
Charlie Conroy $^{1}$, Genevieve J. Graves ${ }^{2}$, And Pieter G. van Dok Kum ${ }^{3}$ \\ Submitted to ApJ
}

\begin{abstract}
The stellar populations of galaxies hold vital clues to their formation histories. In this paper we present results based on modeling stacked spectra of early-type galaxies drawn from the Sloan Digital Sky Survey (SDSS) as a function of velocity dispersion, $\sigma$, from $90 \mathrm{~km} \mathrm{~s}^{-1}$ to $300 \mathrm{~km} \mathrm{~s}^{-1}$. The spectra are of extremely high quality, with typical signal-to-noise ratio, S/N, of $1000 \AA^{-1}$, and a wavelength coverage of $4000 \AA-8800 \AA$. Our population synthesis model includes variation in 16 elements from $\mathrm{C}$ to $\mathrm{Ba}$, a two-component star formation history, the shift in effective temperature, $\Delta T_{\text {eff }}$, of the stars with respect to a solar metallicity isochrone, and the stellar initial mass function (IMF), amongst other parameters. In our approach we fit the full optical spectra rather than a select number of spectral indices and are able to, for the first time, measure the abundances of the elements $\mathrm{V}, \mathrm{Cr}, \mathrm{Mn}, \mathrm{Co}$, and $\mathrm{Ni}$ from the integrated light of distant galaxies. Our main results are as follows: 1) lightweighted stellar ages range from $6-12 \mathrm{Gyr}$ from low to high $\sigma ; 2)[\mathrm{Fe} / \mathrm{H}]$ varies by less than 0.1 dex across the entire sample; 3) $\mathrm{Mg}$ closely tracks $\mathrm{O}$, and both increase from $\approx 0.0$ at low $\sigma$ to $\sim 0.25$ at high $\sigma$; Si and Ti show a shallower rise with $\sigma$, and $\mathrm{Ca}$ tracks $\mathrm{Fe}$ rather than $\mathrm{O}$; 4) the iron peak elements $\mathrm{V}, \mathrm{Cr}, \mathrm{Mn}$, and $\mathrm{Ni}$ track $\mathrm{Fe}$, while Co tracks $\mathrm{O}$, suggesting that Co forms primarily in massive stars; 5) $\mathrm{C}$ and $\mathrm{N}$ track $\mathrm{O}$ over the full sample and $[\mathrm{C} / \mathrm{Fe}]$ and $[\mathrm{N} / \mathrm{Fe}]$ exceed 0.2 at high $\sigma$; and 6) the variation in $\Delta T_{\text {eff }}$ with total metallicity closely follows theoretical predictions based on stellar evolution theory. This last result is significant because it implies that we are robustly solving not only for the detailed abundance patterns but also the detailed temperature distributions (i.e., isochrones) of the stars in these galaxies. A variety of tests reveal that the systematic uncertainties in our measurements are probably 0.05 dex or less. Our derived $[\mathrm{Mg} / \mathrm{Fe}]$ and $[\mathrm{O} / \mathrm{Fe}]$ abundance ratios are $0.05-0.1$ dex lower than most previous determinations. Under the conventional interpretation that the variation in these ratios is due to star formation timescale variations, our results suggest longer star formation timescales for massive early-type galaxies than previous studies. Detailed chemical evolution models are necessary in order to translate the abundance ratio distributions of these galaxies into constraints on their formation histories. Alternatively, these data may provide useful constraints on the nucleosynthetic pathways for elements whose production is not well understood.
\end{abstract}

Subject headings: galaxies: stellar content — galaxies: abundances — galaxies: early-type

\section{INTRODUCTION}

We now have a working theory for the evolution of large scale structure in the universe thanks to the largely successful paradigm of a universe dominated by cold dark matter. In this paradigm cosmological structures form "bottomup" - small structures collapse early and the massive clusters assemble late. One of the more interesting puzzles in this paradigm is the realization that the most massive galaxies in our universe seem to have formed their stars earliest, with lower-mass galaxies forming their stars later (e.g., Trager et al. 1998; Thomas et al. 2005; Jimenez et al. 2007). This phenomenon goes by many names, including "downsizing" and "anti-hierarchical growth". No $a b$ initio model for galaxy formation is able to reproduce these basic features of the observed galaxy population. The apparent tension between the bottom-up nature of cold dark matter and the topdown star formation histories of galaxies can be reconciled by recognizing that the formation time of the stars need not be related to the assembly time of the stars into their present day galaxies.

\footnotetext{
${ }^{1}$ Department of Astronomy \& Astrophysics, University of California, Santa Cruz, CA, USA

${ }^{2}$ Department of Astrophysical Sciences, Princeton University, Princeton, NJ, USA

${ }^{3}$ Department of Astrophysical Sciences, Yale University, New Haven, CT, USA
}

From an empirical point of view, the formation and evolution of galaxies can be probed via two general techniques. The first is through lookback studies where one observes, statistically, the progenitors of present day galaxies at progressively higher redshifts. The second is through studying the present day properties of galaxies, including their stellar populations, structure, and kinematics, in order to learn about their past evolution. This latter technique is often referred to as the archeological approach, and is the subject of this paper.

It has long been appreciated that optical through nearinfrared (NIR) spectra of old stellar systems are rich in absorption features that provide clues to the ages, metallicities, and abundance patterns of the stars (for a review, see Conroy 2013). Since the 1980s the standard analysis technique has been to measure and model the strength of stellar absorption features through the Lick/IDS index system (Burstein et al. 1984: Worthey et al. 1994). Authors typically model a handfull of these indices in order to estimate various stellar population properties.

Analysis of the Lick indices has revealed that early-type galaxies are enhanced in the $\alpha$ element $\mathrm{Mg}$ compared to the abundance patterns of stars in the Galactic disk (Worthey 1994). The $[\alpha / \mathrm{Fe}]$ ratio has long been known to be sensitive to several parameters, including the timescale of star formation, the slope of the initial mass function (IMF) at $>1 M_{\odot}$, the delay time distribution of Type Ia supernovae (SNe), and 


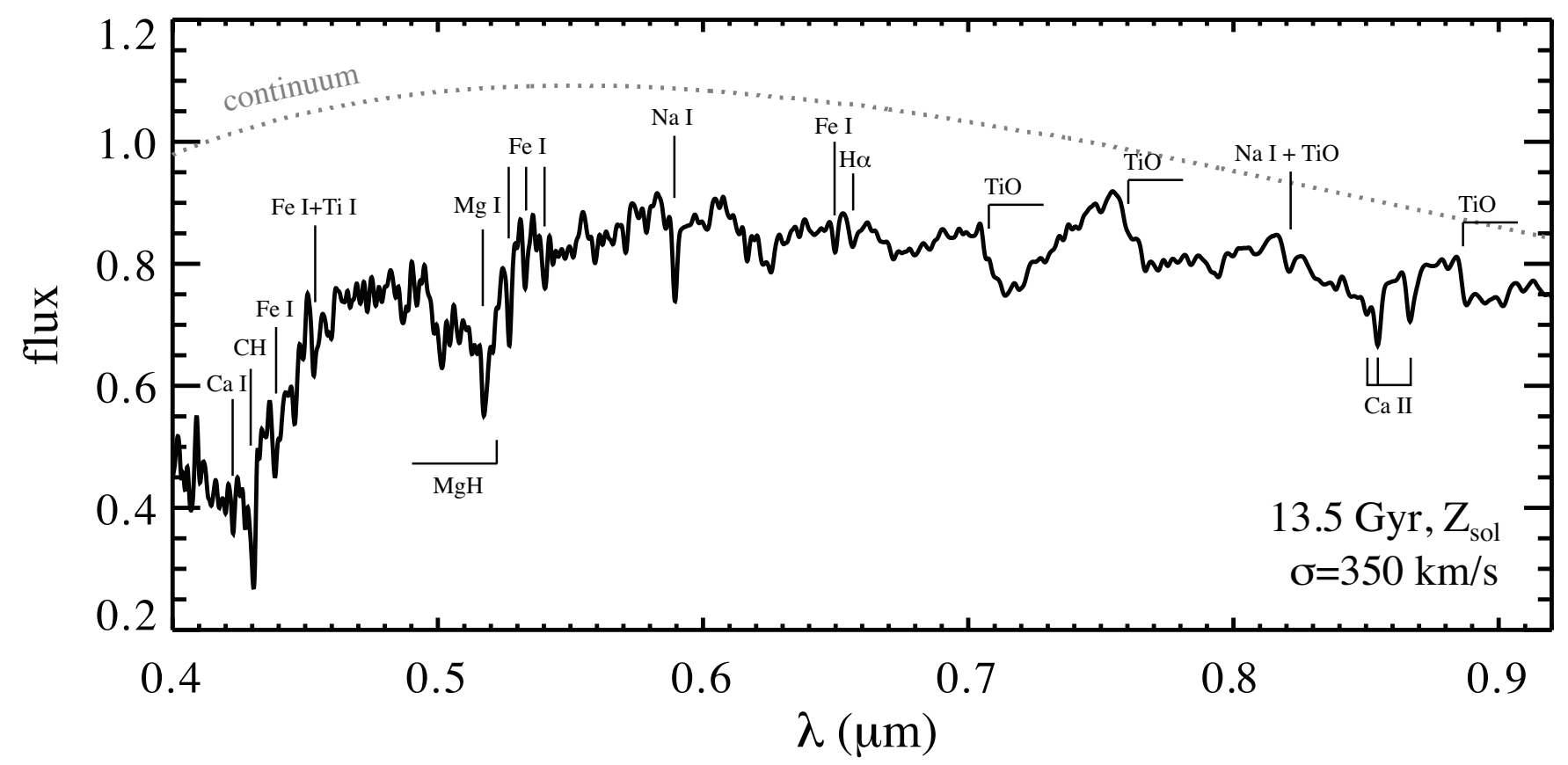

Figure 1. Model spectrum for an age of $13 \mathrm{Gyr}$ and solar metallicity. The spectrum has been smoothed with a velocity dispersion of $\sigma=350 \mathrm{~km} \mathrm{~s}{ }^{-1}$, equal to the smoothing applied to the early-type galaxy data analyzed in this paper. Strong features are labeled. Also included is the location of the true stellar continuum, which is the spectrum that would be observed in the absence of all line opacity. In this figure the model spectrum is computed entirely from synthetic stellar spectra, whereas for the main analysis the synthetic spectra are only used differentially.

the preferential loss of metals via winds (e.g., Tinsley 1979; Thomas et al. 1999). The sensitivity of $[\alpha / \mathrm{Fe}]$ to all of these processes significantly complicates the interpretation of this ratio. Nonetheless, it has become standard practice to assume that the $[\alpha / \mathrm{Fe}]$ ratio is telling us something about the timescale of star formation. By comparing to simple closedbox chemical evolution models, Thomas et al. (2005) concluded that the $[\alpha / \mathrm{Fe}]$ ratios of the most massive galaxies in their sample, with $\sigma \approx 300 \mathrm{~km} \mathrm{~s}^{-1}$, implied star formation timescales of only $\sim 200 \mathrm{Myr}$. These short inferred star formation timescales, if correct, would have profound implications for our understanding of the formation of the most massive galaxies in the universe.

The analysis of spectral indices is beginning to give way to the modeling of the full optical-NIR spectra of stellar systems. This approach has great potential, and is now frequently used to measure star formation histories and metallicities non-parametrically (Heavens et al. 2000; Cid Fernandes et al. 2005; Ocvirk et al. 2006; Tojeiro et al. 2009). Recently, such full spectrum fitting models have been extended to include variation in the elemental abundance patterns (Walcher et al. 2009; Conroy \& van Dokkum 2012a), paving the way to robustly measure many parameters from integrated light spectra.

This paper is part of an ongoing series focused on modeling very high quality optical-NIR spectra of early-type galaxies with full-spectrum fitting stellar population synthesis (SPS) techniques. In Conroy \& van Dokkum (2012a, CvD12), we presented the model, and in Conroy \& van Dokkum (2012b) we used that model to measure the low-mass IMF from the spectra of 34 nearby early-type galaxies and the nuclear bulge of M31 (based on data presented in van Dokkum \& Conroy 2012). In Conroy et al. (2013) we presented results on the neutron-capture elements $\mathrm{Sr}$ and $\mathrm{Ba}$ based on our local galaxy sample and on the stacked spectra that are the focus of this work. In the present paper we measure the ages, detailed abundance patterns, and effective temperatures of the stars in early-type galaxies drawn from the SDSS. In a subsequent paper we will discuss the low-mass IMFs inferred for these galaxies.

The rest of this paper is organized as follows. Section 2 provides an overview of the SPS model and our fitting technique, Section 3 describes the data, and Section 4 presents a test of the model by fitting to spectra of metal-rich star clusters. Our main results are presented in Section [5, and in Section 6 we compare our derived properties to results based on other modeling techniques. A discussion and summary of our results is provided in Sections 7 and 8 .

\section{MODEL \& FITTING TECHNIQUE}

The SPS model used herein was developed in Conroy \& van Dokkum (2012a, CvD12), to which we refer the interested reader for details. The model employs standard SPS techniques, including combining libraries of isochrones and stellar spectra. We use isochrones from three separate groups, all of which are solar metallicity with solar-scaled abundance patterns.

Empirical spectra form the base of the model. We make use of two separate libraries, the MILES library, which covers the wavelength range $0.35 \mu m<\lambda<0.74 \mu m$ (Sánchez-Blázquez et al. 2006), and the IRTF library of cool stars, which covers the wavelength range $0.81 \mu m<\lambda<$ 2.4 $\mu \mathrm{m}$ (Cushing et al. 2005; Rayner et al. 2009). The empirical stars are of approximately solar metallicity and have solar abundance patterns. We have computed a large grid of synthetic stellar atmospheres and spectra in order to model the relative change in the spectrum of a star due to a change in the abundance of a single element. We chose 20 positions along a $13 \mathrm{Gyr}$ isochrone to compute synthetic spectra for each abundance pattern. The model atmospheres and spectra were computed with the ATLAS12 model atmosphere and spectrum synthesis package (Kurucz 1970, 1993), ported to Linux by Sbordone et al. (2004). The line list was provided 

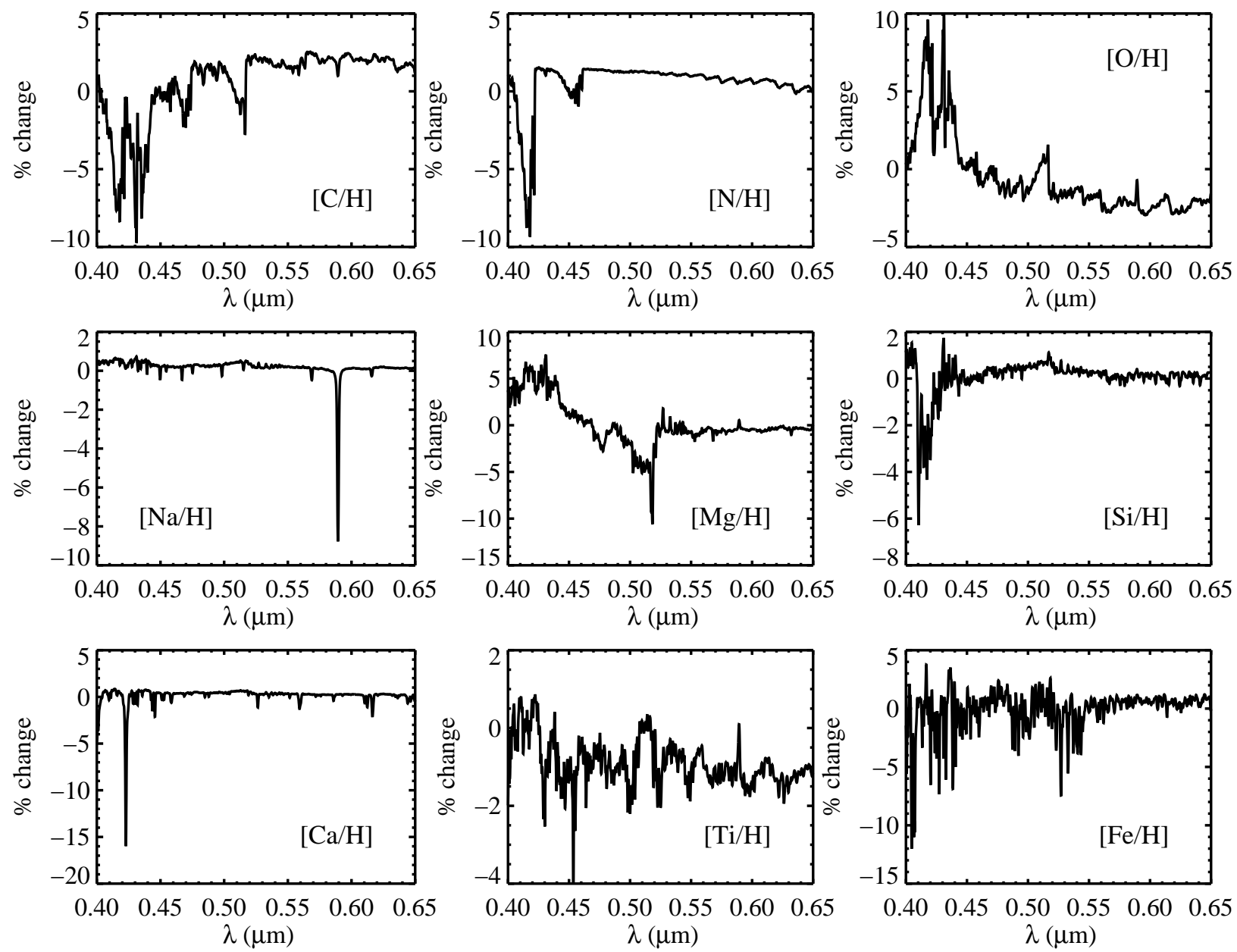

Figure 2. Relative response of the blue spectral region to an increase in the abundance of nine different elements. Abundances have been increased by 0.3 dex for all elements except for C, which is increased by 0.15 dex. The changes are with respect to a solar metallicity model with a MW IMF and an age of 13 Gyr. The models have been smoothed to a velocity dispersion of $\sigma=150 \mathrm{~km} \mathrm{~s}^{-1}$. Notice the different y-axis range in each panel. These are noiseless spectra, and so every feature visible in the figure is real. It is evident from both this figure and Figures 3 and 4 that essentially every spectral region is influenced by one or more elements.

by R. Kuruc4 including linelists for $\mathrm{TiO}$ and $\mathrm{H}_{2} \mathrm{O}$, amongst other molecules. The synthetic stellar spectra were grafted onto the empirical stellar spectra in order to create models with arbitrary abundance patterns. We emphasize that the synthetic spectra are only used differentially. The model allows for arbitrary variation in the IMF and spans ages from $3-13.5$ Gyr. Figure 1 shows the model spectrum for a 13 Gyr, solar metallicity population, smoothed to a velocity dispersion of $\sigma=350 \mathrm{~km} \mathrm{~s}^{-1}$.

There have been several updates to the CvD12 model. Most importantly, the synthetic atmospheres and spectra have been recomputed with an updated line list kindly provided by $\mathrm{R}$. Kurucz. This line list includes many more iron lines and updated wavelengths and hyperfine splitting of many lines. The model now contains variation in many more elements, described below. We have also supplemented the MILES empirical library with stacked SDSS M dwarf spectra from Bochanski et al. (2007). This turns out to be important be-

\footnotetext{
${ }^{4}$ kurucz.harvard.edu/
}

cause the MILES library contains only a few M dwarf spectra, and none cooler than M6V. The SDSS M dwarf template spectra cover the entire $M$ dwarf spectral sequence. The final update since CvD12 is the inclusion of emission lines in the fitting (rather than masking regions of potential emission line contamination, as done previously). Currently we include 13 emission lines, including individual components of [O II] $\lambda 3727,[\mathrm{Ne}$ III] $\lambda 3868, \mathrm{H} \delta, \mathrm{H} \gamma, \mathrm{H} \beta,[\mathrm{O}$ III] $\lambda 5007$, $[\mathrm{N}$ I] $\lambda 5201,[\mathrm{~N} \mathrm{II}] \lambda 6547,6583, \mathrm{H} \alpha$, and [S II] $\lambda 6716,6731$. We include separate parameters for the strengths of each of these lines (i.e., we do not enforce particular line ratios, even amongst doublets with known, constant line ratios). The intrinsic line shape is assumed to be a gaussian with a single width for all lines. This width is also included in the fit.

The effect of varying elemental abundances on the model spectra are shown in Figures 2, 3, and 4. These figures show the response of a $13 \mathrm{Gyr}$, solar metallicity model to an increase in the abundance of a single element. We emphasize that the model atmospheres and spectra are always treated self-consistently (i.e., the elemental concentration is increased 

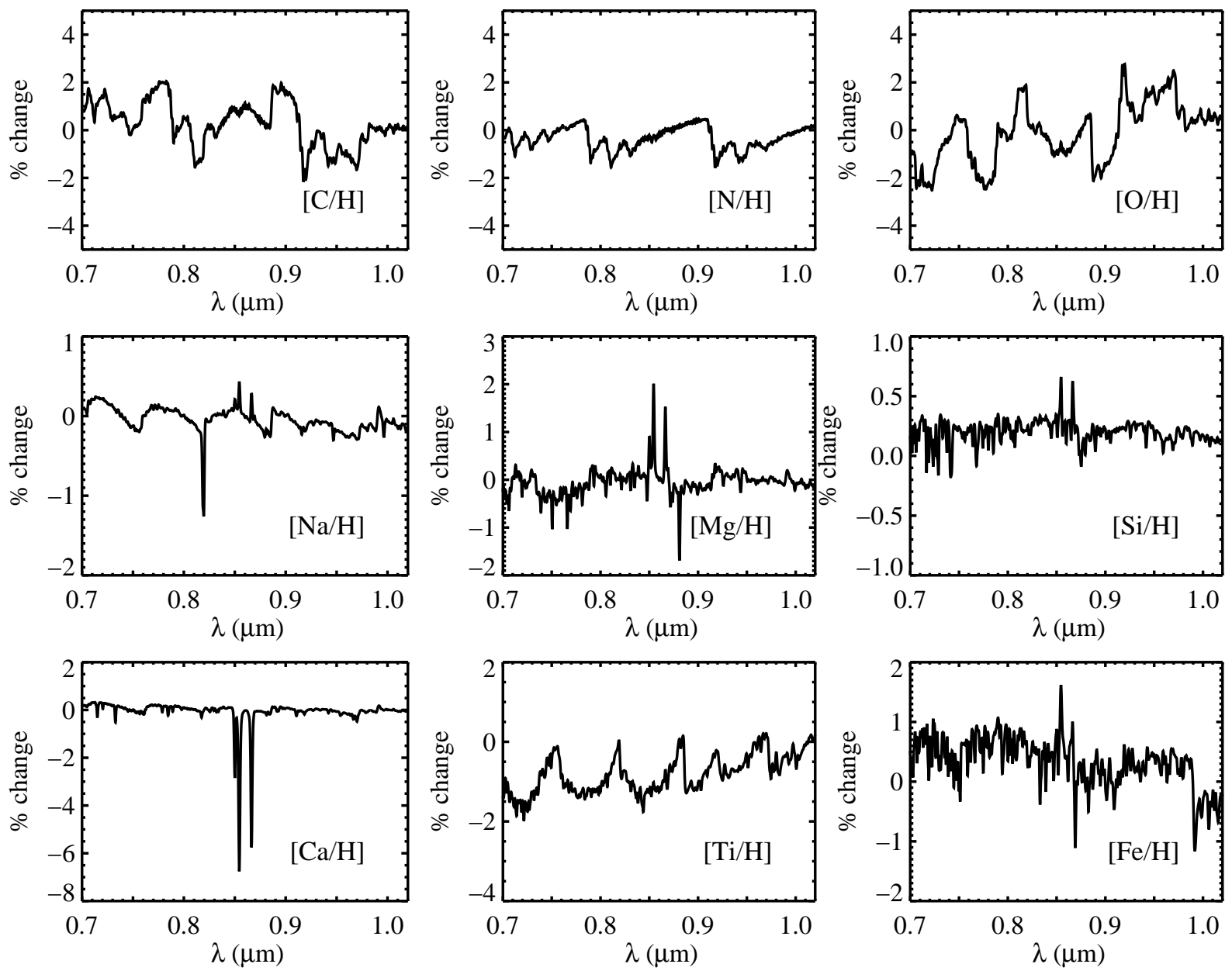

Figure 3. Same as Figure 3 now showing the red spectral region. Abundances have been increased by 0.3 dex for all elements except for C, which is increased by 0.15 dex

both in the computation of the model atmosphere and of the model spectra). In many cases the effect on the spectrum from a change in an element is straightforward, e.g., increasing $[\mathrm{Mg} / \mathrm{H}]$ results in stronger $\mathrm{Mg}$ I lines, but other effects are more subtle. For example, because $\mathrm{Mg}$ is a major electron donor, increasing $[\mathrm{Mg} / \mathrm{H}]$ affects the ionization equilibrium and therefore affects many species of other elements. For example, it is for this reason that $[\mathrm{Mg} / \mathrm{H}]$ affects the strength of the Ca II lines at $\sim 0.86 \mu m$.

The effect of varying age from 7 to 13 Gyr is shown in Figure 5 A variety of temperature-sensitive features are evident, including the hydrogen balmer lines, $\mathrm{NaD}$, and the $\mathrm{Mg}$ I lines. Most importantly, comparison of Figure 5 to Figures 2. 4 shows that when one considers information in the full spectrum, age and metallicity effects are readily separable.

We follow Conroy \& van Dokkum (2012b) in fitting the model to data. In its present form the model contains 40 free parameters, including the redshift and velocity dispersion, a two-part power-law IMF, two population ages (the age of the dominant population and the mass fraction of a $3 \mathrm{Gyr}$ population), four nuisance parameters, 13 emission line strengths, the velocity broadening of the emission lines, and the abundances of $\mathrm{C}, \mathrm{N}, \mathrm{Na}, \mathrm{Mg}, \mathrm{Si}, \mathrm{Ca}, \mathrm{Ti}, \mathrm{V}, \mathrm{Cr}, \mathrm{Mn}, \mathrm{Fe}, \mathrm{Co}, \mathrm{Ni}$,
$\mathrm{Sr}$, and $\mathrm{Ba}$, and $\mathrm{O}, \mathrm{Ne}, \mathrm{S}$ (the latter three are varied in lockstep). These parameters are fit to the data via a Markov Chain Monte Carlo fitting technique. The data and models are split into four wavelength intervals (described below) and, within each interval the spectra are normalized by a high-order polynomial (with degree $n$ where $n \equiv\left(\lambda_{\max }-\lambda_{\min }\right) / 100 \AA$; see Conroy \& van Dokkum 2012b, for details). For the purposes of the present article, the emission line strengths are treated as additional nuisance parameters, and in many cases their strengths are constrained to be very low, which is not surprising given the sample definition described in the next section. Unless specified otherwise, ages quoted in this paper are lightweighted stellar ages computed from the two age components.

One of the nuisance parameters included in the model is a shift in effective temperature, $\Delta T_{\text {eff }}$, applied to all stars used in the synthesis. As described in CvD12, this parameter is meant to capture changes in the isochrones due to changes in abundance patterns of the model stars. The motivation for this shift is shown in Figure 6. In the top panel we show isochrones obtained from the Dartmouth Stellar Evolution Database (DSEP; Dotter et al. 2008) for a range in [Fe/H] and $[\alpha / \mathrm{Fe}]$. In the bottom panel we have shifted each isochrone by a constant amount in $T_{\text {eff }}$ until a match was achieved with the 

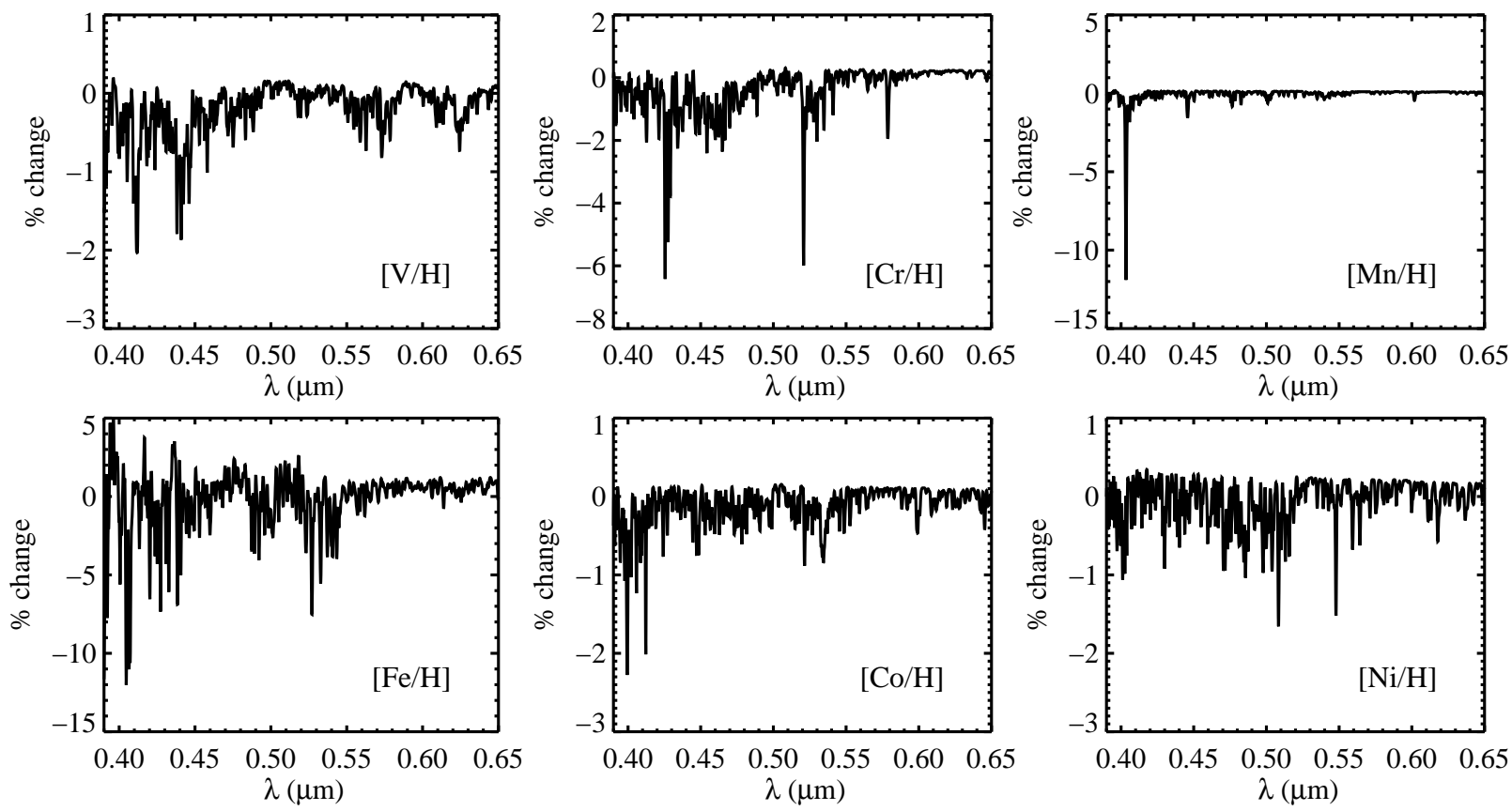

Figure 4. Same as Figure 2 now showing the elements encompassing the iron peak ( $\mathrm{V}, \mathrm{Cr}, \mathrm{Mn}, \mathrm{Fe}, \mathrm{Co}, \mathrm{Ni})$. The change in the model spectra have been computed for a 0.3 dex increase in the abundance of each element.

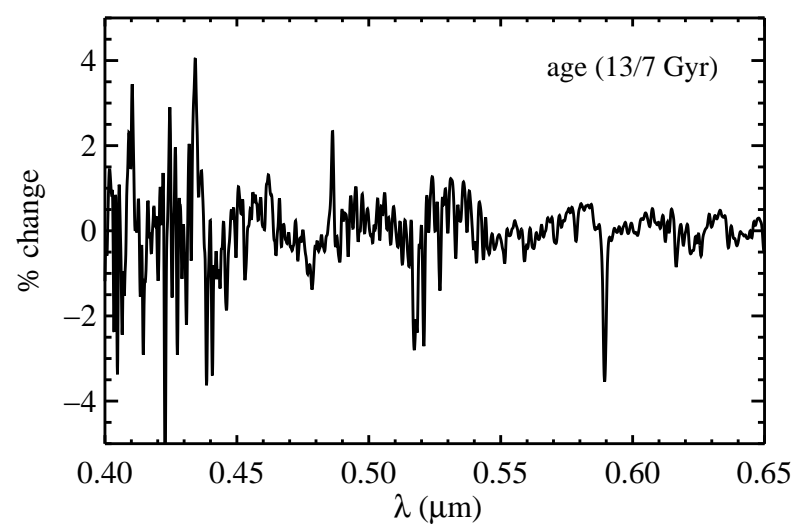

Figure 5. Relative response of the blue spectral region to an age variation from 7 to $13 \mathrm{Gyr}$. The model has solar metallicity and has been smoothed to a velocity dispersion of $\sigma=150 \mathrm{~km} \mathrm{~s}^{-1}$

solar metallicity case. A constant shift does an excellent job of accounting for modest variation in abundance patterns from the solar metallicity model (see also Salaris et al. 1993). We caution that simple shifts in $T_{\text {eff }}$ work less well at low metallicity (VandenBerg et al. 2012). Moreover, it is interesting that the isochrone with $[\mathrm{Fe} / \mathrm{H}]=-0.1$ and $[\alpha / \mathrm{Fe}]=0.2$ is almost identical to the solar metallicity isochrone. Thus, over the metallicity range of interest in this work, a simple shift in $T_{\text {eff }}$ is probably sufficient to account for elemental abundance effects on the isochrones. Because the abundances of many elements are varied simultaneously in the model, we use the free parameter $\Delta T_{\text {eff }}$ to encompass their combined effect, rather than attempting to compute self-consistent isochrones from first principles. However, we caution that a constant shift in $T_{\text {eff }}$ is unlikely to capture the full variation in isochrone morphology with elemental abundance. For example, a change in $[\mathrm{C} / \mathrm{Fe}]$ at fixed $[\mathrm{Fe} / \mathrm{H}]$ results in a slightly cooler main sequence turn-off point and giant branch but a slightly warmer lower main sequence (Dotter et al. 2007; VandenBerg et al. 2012).

\section{DATA}

\subsection{SDSS Data}

The main analysis presented here results from fitting our updated $\mathrm{CvD}$ stellar population model to very high-S/N stacks of passive galaxies, binned by stellar velocity dispersion. These galaxies are selected from the SDSS (York et al. 2000) Main Galaxy Survey (Strauss et al. 2002) Data Release 7 (Abazajian et al. 2009), within a narrow redshift interval $(0.025<z<0.06)$. Following the methodology of Peek \& Graves (2010), we select passive galaxies by requiring that they have no detected emission in $\mathrm{H} \alpha$ nor in [O II] $\lambda 3727$.

The individual spectra have moderate $\mathrm{S} / \mathrm{N}$, typically $\sim 20$ $\AA^{-1}$, so we stack the spectra of hundreds of galaxies to achieve the very high $\mathrm{S} / \mathrm{N}$ needed for detailed abundance analysis. Passive galaxy star formation histories vary strongly as a function of their stellar velocity dispersion, but also at fixed $\sigma$ depending on how far they scatter off the Fundamental Plane (FP; Graves et al. 2010; Springob et al. 2012). We therefore have chosen to stack galaxies in bins of $\sigma$, in order to study the main dimension of stellar population variation, but restrict our sample to galaxies that lie on the FP (i.e., in the central FP slice defined in Graves et al. 2010). The two-dimensional space of galaxy star formation histories will be explored in a future paper.

We stack the spectra in seven bins of velocity dispersion, with mean values of $\sigma=88,112,138,167,203,246$, and $300 \mathrm{~km} \mathrm{~s}^{-1}$. The effective resolution of the data therefore varies from $R \approx 400-1200$ owing to intrinsic Doppler broadening. The SDSS spectra are obtained through $3^{\prime \prime}$ diameter 


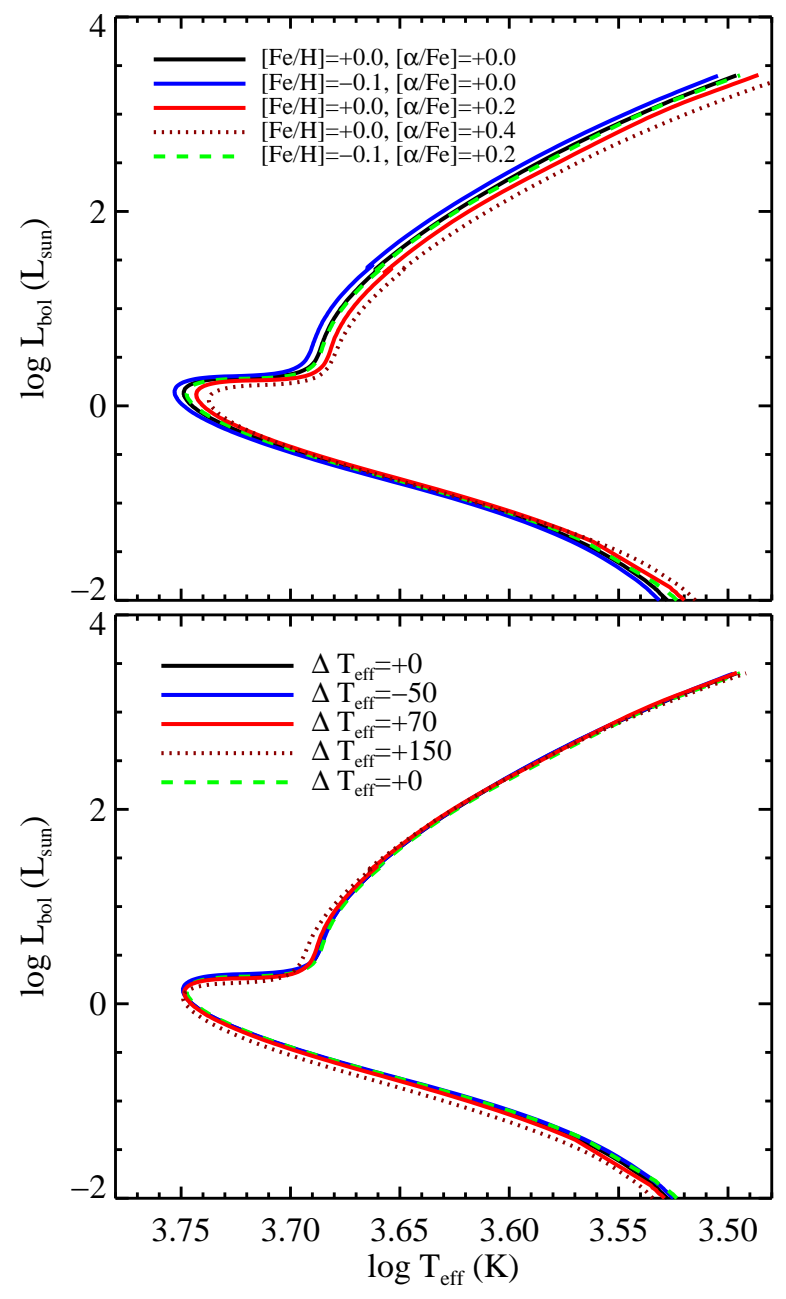

Figure 6. Isochrones at $13.4 \mathrm{Gyr}$ from the Dartmouth Stellar Evolution Database (DSEP) for a variety of chemical compositions. Top panel: Isochrones for a range in compositions approximately spanned by the earlytype galaxies in our sample. The case with $[\mathrm{Fe} / \mathrm{H}]=0.0$ and $[\alpha / \mathrm{Fe}]=+0.4$ is extreme, as no galaxies in the sample have such large $\alpha$ enhancements. Notice that the isochrone with $[\mathrm{Fe} / \mathrm{H}]=-0.1$ and $[\alpha / \mathrm{Fe}]=0.2$ is almost identical to the solar metallicity isochrone. Bottom panel: Same isochrones as in the top panel, with a constant shift in $T_{\text {eff }}$ applied to the entire isochrone (the shift applied is shown in the legend). For the modest range in chemical compositions probed by our sample, the expected effective temperatures of the stars can therefore be well-approximated by a solar metallicity model with a single additional free parameter describing the offset in $T_{\text {eff }}$.

fibers, which sample the inner $\approx 0.8 R_{e}$ for the smallest $\sigma$ bin and the inner $\approx 0.4 R_{e}$ for the largest $\sigma$ bin. Each individual spectrum was continuum-normalized and convolved to an effective dispersion of $350 \mathrm{~km} \mathrm{~s}^{-1}$ before stacking, and each spectrum contributed equally to the stack. Problematic pixels (e.g., those under bright sky lines) were masked before stacking. The resulting stacked spectra have $\mathrm{S} / \mathrm{N}$ at $5000 \AA$ that ranges from $\approx 500 \AA^{-1}$ to $\approx 1800 \AA^{-1}$. Four wavelength intervals were defined for the SDSS stacks, $0.40 \mu \mathrm{m}-0.48 \mu \mathrm{m}$, $0.48 \mu \mathrm{m}-0.58 \mu \mathrm{m}, 0.58 \mu \mathrm{m}-0.64 \mu \mathrm{m}$, and $0.80 \mu \mathrm{m}-0.88 \mu \mathrm{m}$ for fitting models to data.

In Figure 7 we show the stacked spectra in three $\sigma$ bins. The fluxes have been continuum normalized in order to focus
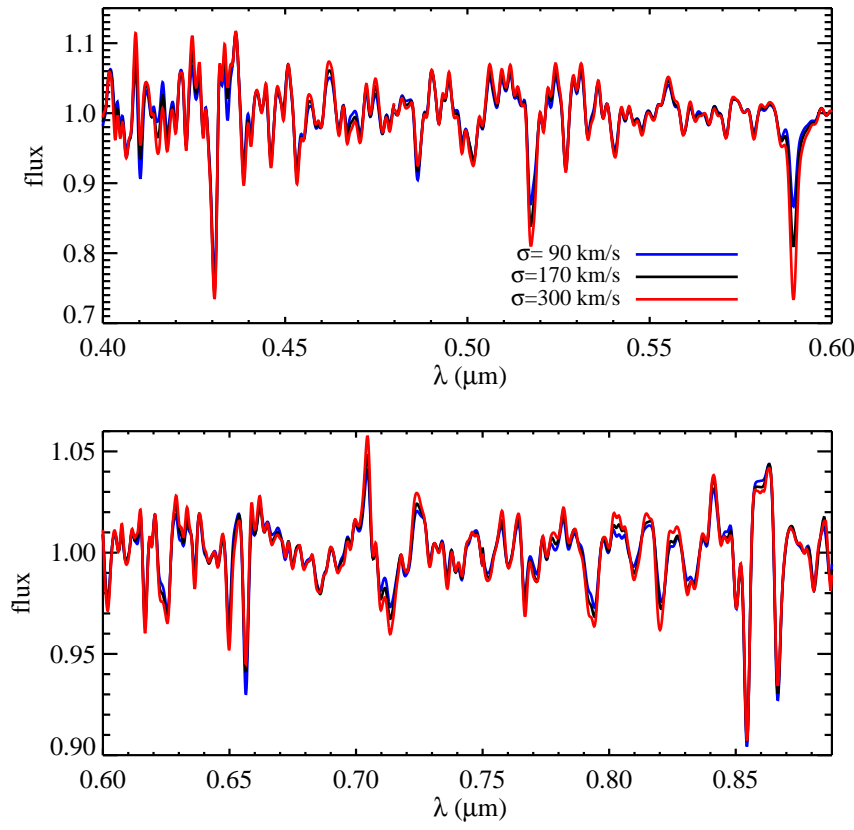

Figure 7. Continuum-normalized stacked spectra of SDSS early-type galaxies in three velocity dispersion bins.

attention on the differences in the line-strengths between different stacks. Notice that the differences are relatively subtle, with features strengths varying by only a few percent from the lowest to the highest dispersion bins.

\subsection{Star Cluster Data}

In this paper we will also analyze integrated light spectra of metal-rich star clusters in order to test the model. We focus on two $\sim Z_{\odot}$ clusters, M67 and NGC 6528. The former is an intermediate-age open cluster with solar-scaled abundance ratios, while the latter is an old bulge globular cluster with enhanced abundance ratios. For M67 we adopt an age of $4 \mathrm{Gyr}$ derived from its color-magnitude diagram (CMD; VandenBerg et al. 2007; Magic et al. 2010), $[\mathrm{Fe} / \mathrm{H}]=0.0$, and solar-scaled abundance ratios with an uncertainty of $0.1 \mathrm{dex}$ (Tautvaišiene et al. 2000; Shetrone \& Sandquist 2000). For NGC 6528 we adopt an age of $11 \mathrm{Gyr}$, consistent with recent CMD-based determinations (Feltzing \& Johnson 2002; Momany et al. (2003). The elemental abundance data are derived from high-resolution spectra of individual RGB stars, as reported in Carretta et al. (2001), Zoccali et al. (2004), and Origlia et al. (2005).

The integrated light spectrum for M67 was constructed in Schiavon et al. (2004) by combining spectra of individual member stars with a Salpeter (1955) IMF. M67 contains a large population of blue straggler stars, but these were omitted in the integrated spectrum. The spectrum covers the range $3640 \AA$, to $5400 \AA$, at a resolution of $2.7 \AA$. The $S / N$ of the integrated spectrum was not provide by Schiavon et al., so we adopt a nominal $\mathrm{S} / \mathrm{N}$ of 100 per pixel.

For NGC 6528 we use the integrated light spectrum from the library of globular cluster spectra presented in Schiavon et al. (2005). These spectra were obtained via drift scan observations, so the spectra reflect the true integrated light of the clusters. The spectrum covers the wavelength range $3360 \AA$ to $6430 \AA$ at a resolution of $\sim 3.1 \AA$. The $\mathrm{S} / \mathrm{N}$ 

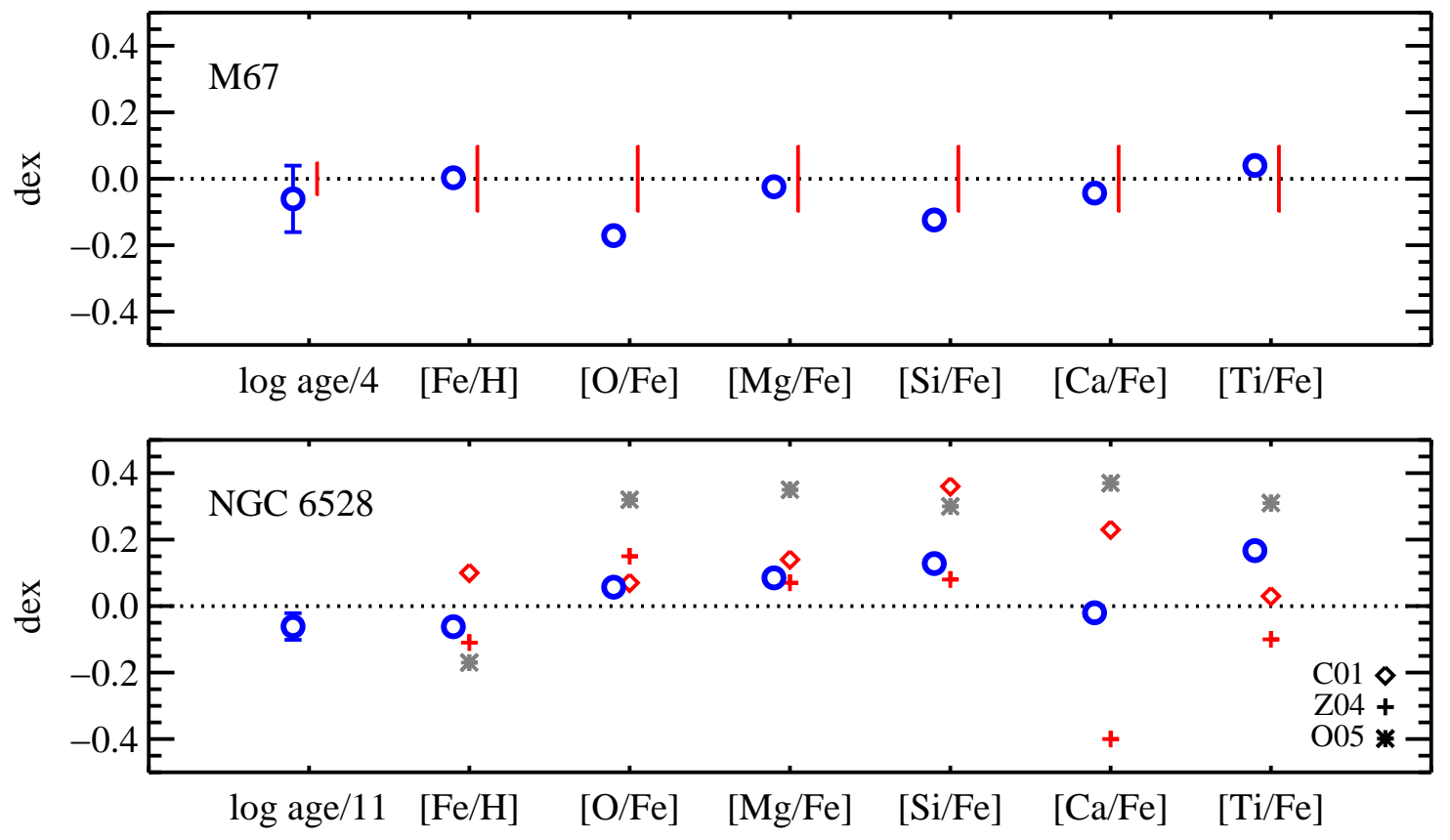

Figure 8. Best-fit parameters for the star clusters M67 (top panel) and NGC 6528 (bottom panel). Results from our full-spectrum fitting of the integrated light (open blue circles) are compared to literature values based on high resolution spectra of individual member stars (red lines and symbols). Fiducial ages for M67 and NGC6528 are taken to be 4 and 11 Gyr, respectively, based on isochrone fitting to their CMDs. Abundance patterns for NGC 6528 are from Carretta et al. (2001, C01), Zoccali et al. (2004, Z04), and Origlia et al. (2005, O05). For M67 we adopt a fiducial abundance pattern of $0.0 \pm 0.1$ (roughly $1 \sigma$ errors Tautvaišiene et al. 2000; Shetrone \& Sandquist 2000). Overall the agreement between our model predictions and the literature values is good, although the large variation in the literature determination of many of the elemental abundances for NGC 6528 prohibits a more definitive test of our model.

Table 1

Results from Modeling Stacked SDSS Early-Type Galaxy Spectra

\begin{tabular}{ccccccccccccccccccc}
\hline \hline $\begin{array}{c}\log \sigma \\
\mathrm{km} \mathrm{s}^{-1}\end{array}$ & $\begin{array}{c}\log M_{*} \\
M_{\odot}\end{array}$ & $\begin{array}{c}\mathrm{rms} \\
\%\end{array}$ & $\begin{array}{c}\text { age } \\
\mathrm{Gyr}\end{array}$ & $\log f_{y}$ & {$[\mathrm{Fe} / \mathrm{H}]$} & $\mathrm{C}$ & $\mathrm{N}$ & $\mathrm{O}$ & $\mathrm{Na}$ & $\mathrm{Mg}$ & $\mathrm{Si}$ & $\mathrm{Ca}$ & $\mathrm{Ti}$ & $\mathrm{V}$ & $\mathrm{Cr}$ & $\mathrm{Mn}$ & $\mathrm{Co}$ & $\mathrm{Ni}$ \\
\hline 1.94 & 9.63 & 0.22 & 6.2 & -0.7 & -0.07 & 0.05 & 0.02 & 0.03 & -0.16 & 0.05 & 0.02 & 0.03 & 0.05 & 0.03 & 0.02 & 0.02 & 0.03 & -0.00 \\
2.05 & 9.75 & 0.22 & 6.5 & -0.9 & -0.05 & 0.07 & 0.08 & 0.06 & -0.09 & 0.08 & 0.00 & 0.03 & 0.07 & 0.01 & 0.00 & 0.03 & 0.06 & -0.00 \\
2.14 & 9.80 & 0.21 & 6.8 & -2.2 & -0.04 & 0.09 & 0.13 & 0.10 & 0.02 & 0.09 & 0.03 & 0.03 & 0.08 & 0.03 & -0.01 & 0.03 & 0.10 & 0.01 \\
2.23 & 10.08 & 0.22 & 7.0 & -4.1 & -0.03 & 0.13 & 0.18 & 0.17 & 0.12 & 0.12 & 0.05 & 0.03 & 0.09 & 0.03 & -0.01 & 0.03 & 0.16 & 0.02 \\
2.30 & 10.55 & 0.26 & 7.6 & -4.3 & -0.01 & 0.16 & 0.21 & 0.20 & 0.22 & 0.15 & 0.09 & 0.03 & 0.11 & 0.03 & -0.03 & 0.02 & 0.20 & 0.03 \\
2.39 & 10.70 & 0.29 & 11.0 & -4.3 & -0.02 & 0.19 & 0.26 & 0.25 & 0.33 & 0.20 & 0.13 & 0.04 & 0.12 & -0.01 & -0.02 & 0.05 & 0.27 & 0.01 \\
2.47 & 11.07 & 0.34 & 11.8 & -4.4 & 0.00 & 0.21 & 0.27 & 0.28 & 0.43 & 0.22 & 0.16 & 0.02 & 0.12 & -0.02 & -0.03 & 0.05 & 0.26 & 0.07
\end{tabular}

Note. - Stellar masses are averages within the $\sigma$ bins and they assume a Kroupa (2001) IMF. The rms deviation between the model and data is computed over the full wavelength range used in the fit. Ages are light-weighted and the fraction of mass in the $3 \mathrm{Gyr}$ component, $f_{y}$, is included in the table. Abundances of elements are quoted as $[\mathrm{X} / \mathrm{Fe}]$. Formal statistical errors are less than $1 \%$ and so are omitted from the table. Systematic errors dominate the error budget, and are estimated to be 0.05 dex or less. See Section 5.4 for further discussion.

ranges from $\approx 50$ per pixel at $4000 \AA$ to 150 per pixel at $5000 \AA$. In this paper we analyze the spectrum extracted within the central $7^{\prime \prime}$. Several spectral regions had to be masked owing to sub-optimal sky subtraction and bad CCD columns. These regions include $4155 \AA-4165 \AA$, $4535 \AA-4565 \AA$, $4850 \AA$ $4870 \AA$, and $5035 \AA$ - $5065 \AA$.

For both spectra we use two wavelength intervals when fitting the model to these data: $4000 \AA-4600 \AA$, and $4600 \AA$ $5350 \AA$.

\section{TESTING THE MODEL AGAINST METAL-RICH STAR CLUSTERS}

Before discussing the main results of this paper, in this section we present the results of fitting our models to spectra of the metal-rich star clusters M67 and NGC 6528. Star clusters are of course simpler to model than galaxies, owing to the fact that they are at least approximately coeval and monometallic. They therefore can serve as useful tests of any SPS model.

In Figure 8 we compare the best-fit ages, $[\mathrm{Fe} / \mathrm{H}]$, and several $\alpha$ elements for these two clusters. Turning first to M67, we derive an age of $3.5 \pm 0.3 \mathrm{Gyr}$, in excellent agreement with the CMD-based age. We also find excellent agreement for $\mathrm{Fe}$, $\mathrm{Mg}, \mathrm{Ca}$, and $\mathrm{Ti}$. The abundances of $\mathrm{O}$ and $\mathrm{Si}$ are underestimated by $1-2 \sigma$. Because the effects of $\mathrm{O}$ and $\mathrm{Si}$ on the spectrum are somewhat more subtle than the other $\alpha$ elements, integrated light spectra that include the redder spectral coverage for M67 are needed before a more definitive conclusion regarding these elements can be reached. Though not shown 

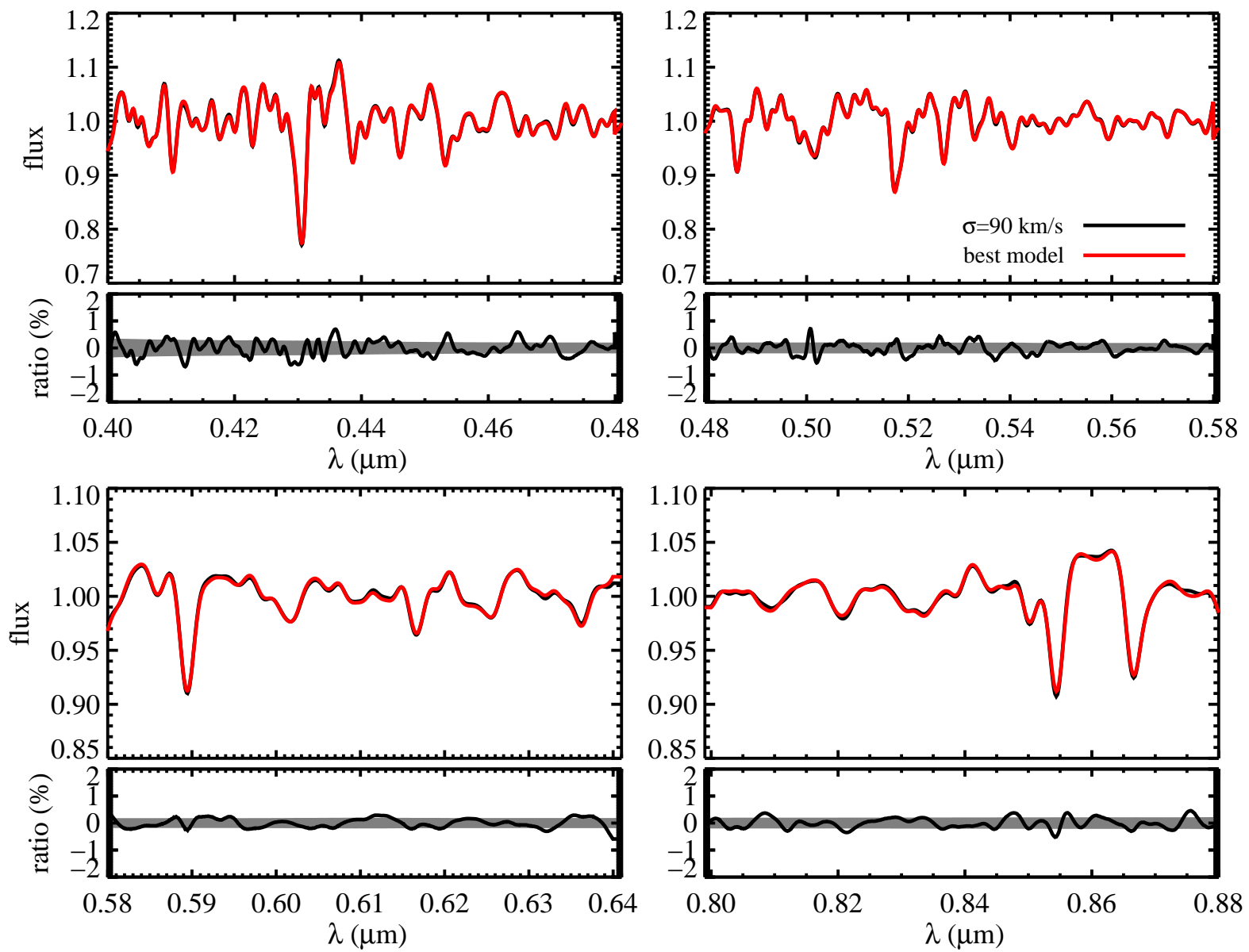

Figure 9. Comparison between the SDSS early-type galaxy stacked spectrum in the lowest velocity dispersion bin (black lines) and the best-fit model (red lines). Within each wavelength range, the top panel shows the continuum-normalized fluxes and the lower panel shows the ratio between data and model. The grey band denotes the $1 \sigma$ error limits of the data. Overall the quality of the fit is excellent.

in Figure 8, we also find $[\mathrm{C} / \mathrm{Fe}]=-0.06$ and $[\mathrm{N} / \mathrm{Fe}]=-0.02$ for M67, which agrees within 0.1 dex with literature determinations of $\mathrm{C}$ and $\mathrm{N}$ from main sequence turn-off stars in M67 (Shetrone \& Sandquist 2000).

The model also provides an excellent fit to the age and $[\mathrm{Fe} / \mathrm{H}]$ abundance of NGC 6528. The $\alpha$ elements also broadly agree with the literature data, although perhaps the most striking impression from Figure 8 is the large scatter between the literature estimates for the abundance ratios. The most dramatic difference is in the estimated $[\mathrm{Ca} / \mathrm{Fe}]$ abundance, where Origlia et al. (2005) find $[\mathrm{Ca} / \mathrm{Fe}]=+0.4$ while Zoccali et al. (2004) quote $[\mathrm{Ca} / \mathrm{Fe}]=-0.40$; a difference of 0.8 dex! We have plotted the Origlia et al. (2005) results in grey because these authors model high-resolution NIR spectra while Carretta et al. (2001) and Zoccali et al. (2004) model high-resolution blue spectra. We also draw attention to the fact that these two authors find relatively modest enhancements in $\mathrm{O}$ and $\mathrm{Mg}$, less than 0.2 dex. Thus, while NGC 6528 is $\alpha$-enhanced, it is not tremendously so.

For NGC 6528 we do not compare results for $\mathrm{C}$ nor $\mathrm{N}$ because these elements can be synthesized in the envelopes of red giant branch (RGB) stars, so abundances of these elements derived from such stars do not reflect the original (i.e., main sequence) abundances. Our model recovers the latter, whereas literature estimates of $\mathrm{C}$ and $\mathrm{N}$ for this cluster are based on RGB stars. Owing to the large discrepancy in reported literature abundances, even for important species such as $\mathrm{Fe}, \mathrm{O}$, $\mathrm{Mg}, \mathrm{Si}, \mathrm{Ca}$, and $\mathrm{Ti}$, we made no effort to compare to other elements as their derived abundances from high-resolution stellar spectroscopy appear to be even more uncertain.

Our model successfully recovers the ages, $[\mathrm{Fe} / \mathrm{H}]$, and $\alpha$ element abundances of metal-rich star clusters. Further progress on testing SPS models must await 1) higher quality integrated light spectra of star clusters covering a wider wavelength range and at higher $\mathrm{S} / \mathrm{N}$ than is presently available; 2) a thorough evaluation of the results from high-resolution stellar spectroscopy. Until a consensus emerges on the abundance patterns of the stars in metal-rich star clusters, such systems cannot be used to rigorously test abundance ratio predictions of SPS models.

\section{RESULTS}

We now turn to our main results - the analysis of high quality stacked spectra of early-type galaxies from the SDSS. In this section we present and discuss the overall quality of the fits, the detailed abundance patterns, and the derived effective 

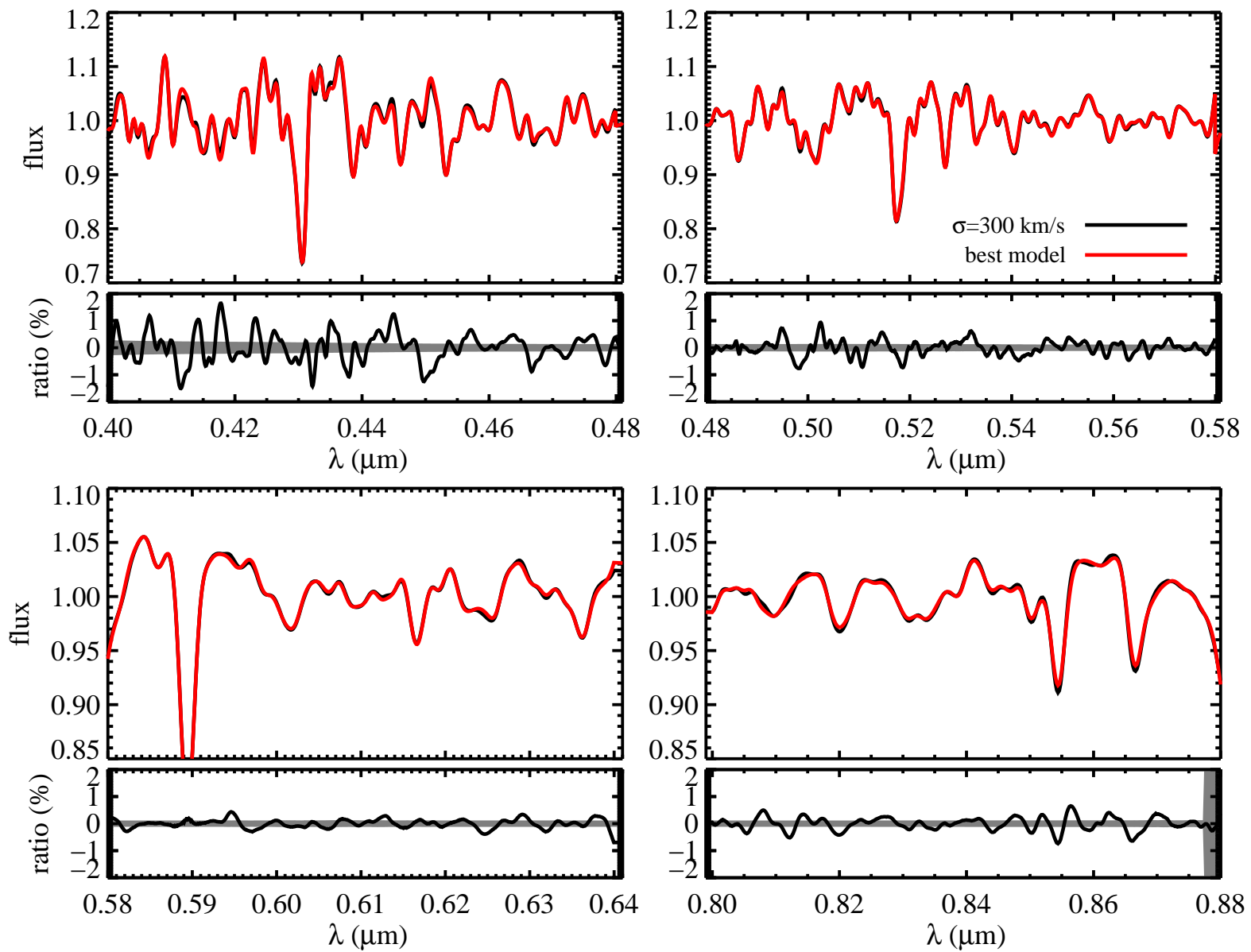

Figure 10. Same as Figure 9 now showing results for the highest velocity dispersion bin. As in Figure 9 the overall quality of the fit is excellent.

temperatures of the stars. Table 1 presents the main derived parameters from modeling the stacked spectra. Results for the abundance ratios of neutron capture elements $\mathrm{Sr}$ and $\mathrm{Ba}$ were presented in Conroy et al. (2013), and the results for the variation in the IMF with galaxy $\sigma$ will be presented in future work. We simply note here that the IMF trends with $\sigma$ are similar to those presented in Conroy \& van Dokkum (2012b). We include in this table estimated stellar masses based on the mean $r$-band luminosities of the galaxies in each bin combined with the derived stellar $M / L$ values assuming a Kroupa (2001) IMF.

\subsection{Overall Quality of the Fits \& Uniqueness of the Derived Parameters}

We begin by discussing the overall quality of the model fits. In Figures 9 and 10 we show the data and best-fit model for the lowest and highest $\sigma$ bins. Within each panel the fluxes have been continuum-normalized by a high-order polynomial. These figures demonstrate the overall very high quality of the fits. In the lowest dispersion bin the rms between data and model is only $0.22 \%$, and $\chi^{2} / \mathrm{dof}=1.1$, indicating that our model is able to fully capture the observed variation in the spectrum to the precision allowed by the data. At the highest dispersion bin, where the abundance ratios deviate most strongly from the solar-scaled values, the rms is $0.34 \%$, with $\chi^{2} / \mathrm{dof}=4.7$. It is not surprising that the fit is formally worse for the highest dispersion bin, both because the $\mathrm{S} / \mathrm{N}$ is higher, which imposes more stringent demands on the model, and because the strong deviations from solar-scaled abundance ratios means that the synthetic stellar spectra play an important role in the modeling. The synthetic spectra carry significant uncertainties (e.g., due to the treatment of the model atmospheres and incomplete line lists), so their greater weight in the model will tend to result in larger model uncertainties.

In Figure 11 we show the residuals now for all seven bins. The most striking feature of the residuals is their similarity across the sample. For example, the residuals around the Ca II triplet at $8500 \AA-8700 \AA$ are relatively significant, but the magnitude of the residuals does not vary strongly with galaxy velocity dispersion (see also Conroy \& van Dokkum 2012b, where this was demonstrated on an object-by-object basis). This weak dispersion-dependence in the residuals is important for several reasons. First and foremost, it suggests that the trends in the derived parameters, to be discussed later in this section, are likely insensitive to remaining model systematics. In addition, the fact that the residuals at high dispersion are similar to the lowest dispersion bin, where the abundance ratios are close to solar-scaled values, suggests that the model deficiencies lie in the base models constructed with empirical 

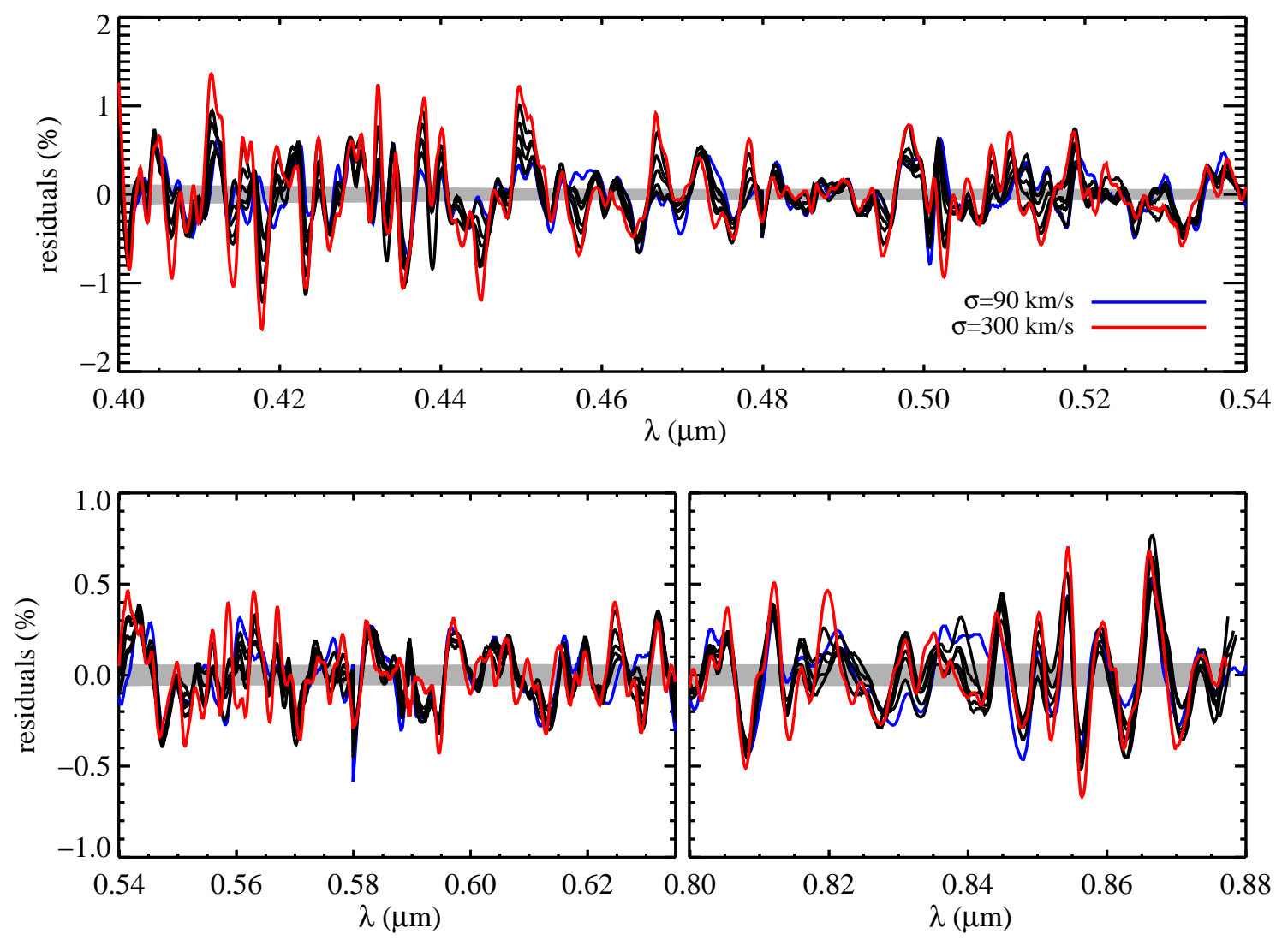

Figure 11. Residuals between the data and best-fit models for all seven stacked early-type galaxy spectra analyzed in this paper. The shaded region marks the noise limit of the middle $\sigma$ bin. In most spectral regions the residuals are constant across the sample, which is an important result because it means that the higher $\sigma$ galaxies, which harbor abundance patterns significantly different from the solar neighborhood, are nearly as well described by our model as the lower $\sigma$, less metal-enhanced galaxies. In a few spectral regions, e.g., at $0.45 \mu \mathrm{m}$ the residuals correlate with galaxy velocity dispersion, indicating that our model is not capturing the totality of variation in the observed spectra.

stellar spectra. It is worth pointing out that the MILES stellar library, which forms the core of the model in the optical, has typical S/N of $\sim 150 \AA^{-1}$, so at the level of interest here the uncertainties in the empirical spectra probably play an important role (the residuals in Figure 11 are smooth in part because the model and data have been smoothed to $\sigma=350 \mathrm{~km} \mathrm{~s}^{-1}$ ).

Some systematics with dispersion are evident, for example at $4500 \AA$ and $4675 \AA$. These are probably due to features that are not well-captured in our synthetic spectral library. In future work we will investigate the sources of line opacity in these wavelength regions in an attempt to further improve the models.

It is also apparent from Figure 11 that the residuals are larger in the blue than in the red. This is likely due to the fact that the blue spectral region contains more and stronger atomic and molecular absorption lines than the red (e.g., compare Figures 2 and 3). Galaxies with non-solar abundance patterns will therefore place more demanding requirements on our models in the blue compared to the red. It is also evident that the residuals in the blue are much more dependent on the galaxy velocity dispersion than in the red. In the red spectral region the median absolute residuals are approximately constant with $\sigma$ at $0.1 \%$ while in the blue they increase from $0.16 \%$ at low $\sigma$ to $0.25 \%$ at high $\sigma$ (cf. Figures 9 and 10 ).

Our model contains 40 free parameters that are fit to the data via an MCMC algorithm. The parameter space is very large and it is difficult with any algorithm to ensure that one has reached the true global maximum of the likelihood surface. Nonetheless, we have performed a large number of tests, including re-starting the chains at random locations in parameter space. Each time the chain re-converges to the same maximum. Moreover, tests have demonstrated that the likelihood surface is very smooth, especially for the high $\mathrm{S} / \mathrm{N}$ data we are modeling, and so for this reason isolated local maxima appear to be rare.

We can also explore the level of covariance (degeneracy) between various parameters. This was discussed in Conroy \& van Dokkum (2012b); we revisit this issue here in a different context. In Figure 12 we plot the covariance between the abundances of $\mathrm{C}, \mathrm{O}$, and $\mathrm{Ti}$, as the abundances of three elements may on physical grounds be expected to display some degree of covariance. For example, inspection of Figures 2 and 3 demonstrates that the response functions of these elements are similar in several respects. This similarity is driven in part by molecular equilibrium involving $\mathrm{CO}$ and TiO. But from Figure 12 it is evident that our model is capable of separately measuring these three quantities with very high accuracy and modest levels of degeneracy (basically no degeneracy at $68 \%$ confidence and a moderate degree of degeneracy at the $95 \%$ confidence limit for $[\mathrm{O} / \mathrm{Fe}]$ vs. $[\mathrm{C} / \mathrm{Fe}]$, as might be expected from Figure 2). We have inspected a wide variety of other correlations and find in all cases the level of 


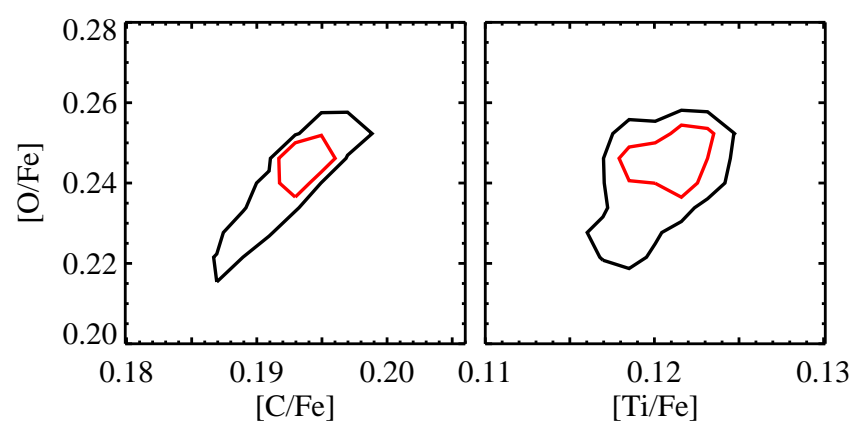

Figure 12. Covariance between $[\mathrm{O} / \mathrm{Fe}],[\mathrm{C} / \mathrm{Fe}]$ and $[\mathrm{Ti} / \mathrm{Fe}]$ for the SDSS stacked spectrum with $\log \sigma=2.39 \mathrm{~km} \mathrm{~s}^{-1}$. Contours show $68 \%$ and $95 \%$ confidence limits in the posterior distribution. The response function due to $\mathrm{O}$ variation is similar (though opposite in sign) to $\mathrm{C}$ variation at $\lambda<6000 \AA$ (see Figure 2, which is mostly a consequence of molecular equilibrium involving $\mathrm{CO}$. Similarly, in the red, the response functions of $\mathrm{O}$ and $\mathrm{Ti}$ resemble one another, here because of TiO. This figure demonstrates that, in the context of our model, we are able to separately constrain $\mathrm{C}, \mathrm{O}$, and Ti very well (notice the small range of the $x$ and $y$-axes).

degeneracy between parameters to be very small. When fitting spectra with very high $\mathrm{S} / \mathrm{N}$ there is apparently sufficient information to strongly constrain at least 40 free parameters. In this regime it is the systematic uncertainties that dominate the error budget, the magnitude of which we explore in Section 5.4 .

\subsection{Abundance Patterns}

We now turn to the elemental abundance ratios derived from our sample of stacked SDSS early-type galaxy spectra.

In Figure 13 we show the relations between velocity dispersion and $\mathrm{C}$ and $\mathrm{N}$ (left panel), and the $\alpha$ elements $\mathrm{O}, \mathrm{Mg}, \mathrm{Si}$, $\mathrm{Ca}$, and $\mathrm{Ti}$ (right panel). In most cases the formal statistical errors are smaller than the symbol sizes. The abundance of both $\mathrm{C}$ and $\mathrm{N}$ increase from $[\mathrm{C} / \mathrm{Fe}] \approx[\mathrm{N} / \mathrm{Fe}] \approx 0.0$ at low $\sigma$ to $0.2-0.3$ at high $\sigma$. This is in qualitative agreement with previous results (Kelson et al. 2006; Graves et al. 2007; Schiavon 2007; Smith et al. 2009; Johansson et al. 2012).

The derived abundance ratio patterns of the $\alpha$ elements show several interesting features. First, all ratios are close to the solar-scaled values at low $\sigma$. Second, the trends with $\sigma$ are stronger for the lighter elements $\mathrm{O}$ and $\mathrm{Mg}$ compared to the heavier elements $\mathrm{Si}, \mathrm{Ca}$, and $\mathrm{Ti}$. $\mathrm{Mg}$ appears to track $\mathrm{O}$ closely, with a slight preference for $[\mathrm{Mg} / \mathrm{O}]<0.0$ at high $\sigma$. Ca tracks Fe closely over the full sample, in agreement with previous work (Saglia et al. 2002; Cenarro et al. 2003; Thomas et al. 2003a; Graves et al. 2007; Schiavon 2007; Smith et al. 2009; Johansson et al. 2012). This suggests that a substantial amount of $\mathrm{Ca}$ is formed in Type Ia SNe, as expected from theoretical yields (Nomoto et al. 1984). The enhancement of $\mathrm{Si}$ and $\mathrm{Ti}$ at high $\sigma$ also broadly agrees with earlier work (Milone et al. 2000; Johansson et al. 2012; Worthey et al. 2013).

In Figure 14 we show the results for $[\mathrm{Fe} / \mathrm{H}]$ in addition to the other iron peak elements $\mathrm{V}, \mathrm{Cr}, \mathrm{Mn}, \mathrm{Co}$, and $\mathrm{Ni}$, all of which are measured here for the first time. The $[\mathrm{Fe} / \mathrm{H}]$ abundance increases modestly over the sample by only 0.07 dex. This remarkable uniformity of the Fe abundance, in light of the substantial variation in the abundance of the light and $\alpha$ elements, poses a challenge to chemical evolution models of early-type galaxies. The abundances of the iron peak elements $\mathrm{V}, \mathrm{Cr}, \mathrm{Mn}$, and Ni closely track Fe over the full sample, implying that these elements form in the same nucleosynthetic sites. In stark contrast is $\mathrm{Co}$, which increases in relative abundance to $\mathrm{Fe}$ as $\sigma$ increases, reaching $[\mathrm{Co} / \mathrm{Fe}] \approx 0.27$ at the highest dispersions.

This result is explored further in Figure 15, where we show the abundance ratios of several elements with respect to $\mathrm{O}$ as a function of $\sigma$. Clearly both $\mathrm{N}$ and Co track $\mathrm{O}$ remarkably closely. $\mathrm{C}$ also tracks $\mathrm{O}$ more closely than $\mathrm{Fe}$, but $[\mathrm{C} / \mathrm{O}]$ is not quite as constant as $[\mathrm{N} / \mathrm{O}]$ and $[\mathrm{Co} / \mathrm{O}]$, suggesting that $\mathrm{C}$ and $\mathrm{N}$ may not be forming in exactly the same environments.

Although not shown in a figure, the $[\mathrm{Na} / \mathrm{Fe}]$ abundance also varies considerably across the sample, from -0.16 to 0.43 , qualitatively tracking the light $\alpha$ elements (see Table 11). In detail however the $\mathrm{Na}$ abundance variation is considerably stronger than the other elements. The only two $\mathrm{Na}$ lines available to us are the Na I features at $5895 \AA$ and $8190 \AA$, respectively. The former is well-known to be affected by the presence of an interstellar medium (ISM). However, the sample was selected to have no detectable emission in $\mathrm{H} \alpha$ nor in [O II], suggesting that contamination by ISM absorption should be small. Moreover, one might have expected ISM contamination to be greater at lower $\sigma$, because low mass galaxies are younger, and yet the derived $[\mathrm{Na} / \mathrm{Fe}]$ at low $\sigma$ is lower than either $[\mathrm{O} / \mathrm{Fe}]$ or $[\mathrm{Mg} / \mathrm{Fe}]$ (the presence of an ISM would cause stronger absorption at $5895 \AA$ and thus larger derived $[\mathrm{Na} / \mathrm{Fe}]$ values). Of course, it is not necessary that $[\mathrm{Na} / \mathrm{Fe}]$ track the other elements in detail, as its nucleosynthetic origins are quite complex, including contributions from both massive stars and intermediate-mass asymptotic giant branch (AGB) stars. Indeed, the chemical evolution model of Arrigoni et al. (2010) finds a variation in $[\mathrm{Na} / \mathrm{Fe}]$ in broad agreement with what we find herein, ranging from -0.2 at low mass to +0.3 at high mass.

\subsection{Effective Temperatures}

As discussed in Section 2, one of the free parameters in our model is the shift in $T_{\text {eff }}$ from a fiducial solar metallicity isochrone. This is an important parameter to include because, as we saw in the previous section, the metallicities and abundance patterns vary by factors of several across our sample. A change in metallicity and/or abundance pattern will induce a change in $T_{\text {eff }}$ of the stars (Dotter et al. 2007). It would be difficult to include this variation self-consistently owing to the large grid of isochrones that would be required. The parameter $\Delta T_{\text {eff }}$ is meant to capture all of these effects into a single number.

In Figure 16 we show the resulting relation between $\Delta T_{\text {eff }}$ and the sum of $[\mathrm{O} / \mathrm{Fe}]$ and $[\mathrm{Fe} / \mathrm{H}]$, which is a proxy for $[\mathrm{Z} / \mathrm{H}]$ (e.g., Trager et al. 2000). The relation derived for our sample of SDSS early-type galaxies is compared to theoretical expectations from the Dartmouth isochrones (Dotter et al. 2008). For the latter, all of the $\alpha$ elements track each other. The early-type galaxy sample increases in $\sigma$ from low to high metallicity. The theoretical predictions are based on the models shown in Figure 6. The star symbol corresponds to the model with $[\mathrm{Fe} / \mathrm{H}]=-0.1$ and $[\alpha / \mathrm{Fe}]=+0.2$ and highlights the fact that $\Delta T_{\text {eff }}$ is not a simple function of $[\mathrm{Z} / \mathrm{H}]$.

The excellent agreement in the slope of the $\Delta T_{\text {eff }}$ vs. $[\mathrm{O} / \mathrm{Fe}]+[\mathrm{Fe} / \mathrm{H}]$ relation between the theoretical prediction and the data suggests that we are reliably measuring the distribution of effective temperatures for the stars in these galaxies (i.e., we are solving for the detailed shape of the isochrones). The flattening of the observed trend at high $\sigma$ is interesting and will be explored in detail in future work. The offset between the two relations is $\approx 40 \mathrm{~K}$, which is well within both 

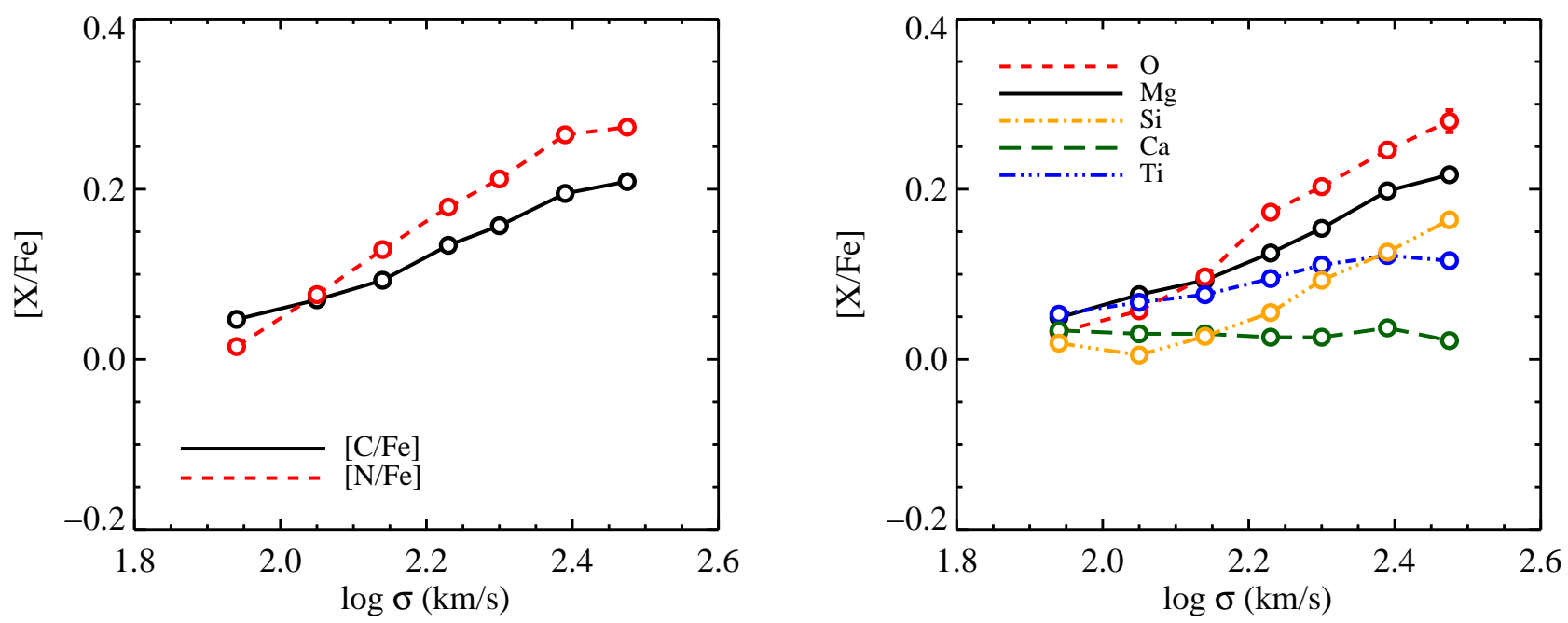

Figure 13. Abundance ratios of $\mathrm{C}, \mathrm{N}$, and the $\alpha$ elements as a function of early-type galaxy velocity dispersion. In most cases the statistical errors are smaller than the symbol sizes. C, N, O, and Mg all seem to track each other fairly closely, while the heavier $\alpha$ elements $\mathrm{Si}$, Ca, and Ti show weaker trends with $\sigma$. Ca clearly tracks Fe instead of O. Systematic errors are probably $<0.05$ dex (see Section 5.4).
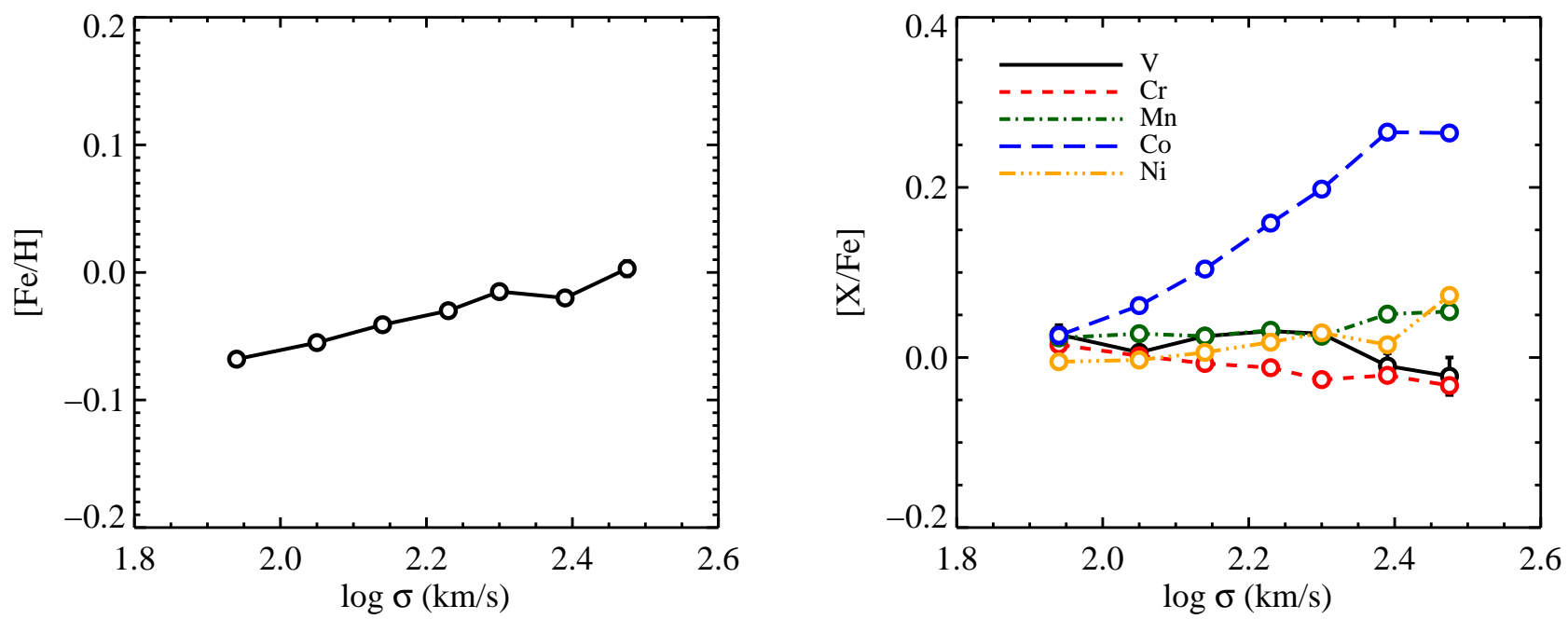

Figure 14. Abundance ratios of the iron peak elements as a function of early-type galaxy velocity dispersion. In most cases the statistical errors are smaller than the symbol sizes. All iron peak elements track Fe except for $\mathrm{Co}$, which seems to track the light $\alpha$ elements $\mathrm{O}$ and $\mathrm{Mg}$. Systematic errors are probably $<0.05$ dex (see Section 5.4).

the systematic uncertainties in the color-temperature relations used to assign temperatures to empirical stars and the theoretical uncertainties in modeling RGB temperatures. In order to provide a more direct comparison between the observations and the theoretical predictions we will need to create isochrones with the exact same chemical composition as we derive for the early-type galaxies (e.g., variation in $\mathrm{C}$ and $\mathrm{N}$ in addition to the $\alpha$ elements). This work is ongoing.

We remind the reader that a multitude of parameters are varying simultaneously across the sample, and so it is impossible at present to cleanly separate correlation from causation. The correlation seen in Figure 16 could be driven by a third variable, and the possibility remains that the $\Delta T_{\text {eff }}$ parameter is soaking up an unidentified model deficiency that varies with $\sigma$ and hence $[\mathrm{Z} / \mathrm{H}]$.

\subsection{Exploration of Systematic Uncertainties}

In this section we explore variations in the fiducial model and in our primary set of stacked spectra in order to quantify systematic uncertainties in our main results. In Figure 17 we plot the difference in either abundance ratio or log(age) between our main results and a modification to our standard analysis. In the upper left panel we compare our standard analysis to a simplified model with fewer free parameters. In particular, in this model the IMF is fixed to the Kroupa (2001) form, only a single age component is included (rather than the standard two components), the iron peak elements are forced to track Fe, and all the nuisance parameters are turned off (including $\left.\Delta T_{\text {eff }}\right)$. This model results in a larger rms residual between data and model compared to our fiducial model (i.e., it is a poorer fit), which should not be surprising given that there are fewer free parameters. Nonetheless, the resulting 
abundance ratios agree well with the standard model, in particular $\mathrm{Mg}, \mathrm{C}, \mathrm{Ca}, \mathrm{Si}, \mathrm{Ti}$, and $\mathrm{Fe}$ agree to within 0.1 dex and in most cases to within 0.05 dex. The largest outliers are the age at low $\sigma$ and the $[\mathrm{O} / \mathrm{Fe}]$ and $[\mathrm{N} / \mathrm{Fe}]$ abundances. The difference in $[\mathrm{O} / \mathrm{Fe}]$ is particularly strong. We speculate that the larger range in $[\mathrm{O} / \mathrm{Fe}]$ in the simple model is due to the fact that the parameter $\Delta T_{\text {eff }}$ is not included. In essence, $[\mathrm{O} / \mathrm{Fe}]$ is forced to vary more in order to compensate for the lack of variation in $\Delta T_{\text {eff }}$.

In the upper right panel we consider a model where the mean wavelength-dependent residuals averaged over the sample are added back into the model. In other words, in this case the model is multiplied by the mean residuals shown in Figure 11 before the model is fit to the data. This will obviously result in a much lower rms difference between the best-fit model and data, and the question is whether or not this changes the derived parameters. As demonstrated in Figure 17, the resulting change in abundance patterns is very small, typically $<0.03$ dex. This implies that the small mis-match between the data and our model does not bias the resulting best-fit parameters.

In the lower left panel we consider the change in parameters when only the blue spectral region $(\lambda<5800 \AA)$ is included in the fit. Here again most parameters change very little, by $<0.05$ dex. Significant outliers include $[\mathrm{Na} / \mathrm{Fe}]$ and $[\mathrm{O} / \mathrm{Fe}]$. The change in $[\mathrm{Na} / \mathrm{Fe}]$ is not surprising because the two features most sensitive to $\mathrm{Na}$ are the $\mathrm{Na}$ I features at $5895 \AA$ and $8190 \AA$. With these features masked out the constraint on the $\mathrm{Na}$ abundance is coming exclusively from the effect of $\mathrm{Na}$ on the electron pressure in the stellar atmospheres, which indirectly affects the ionization states of other elements. For $[\mathrm{O} / \mathrm{Fe}]$ the deviation is also significant, exceeding $0.1 \mathrm{dex}$ in several bins. The origin of this offset is not clear, although we note that the best-fit $\Delta T_{\text {eff }}$ parameter is lower in the case of fitting only the blue spectra. With cooler stars the metal-lines are stronger, perhaps alleviating the need for higher $[\mathrm{O} / \mathrm{Fe}]$ values. This highlights the need for spectra that extend into the red, where the $\Delta T_{\text {eff }}$ parameter has a large effect and hence can be more robustly measured. Moreover, at $\lambda<5800 \AA$ the constraint on $[\mathrm{O} / \mathrm{Fe}]$ is coming primarily through the effect of $\mathrm{O}$ on molecular dissociation equilibrium in the stellar atmospheres, while at $\lambda>5800 \AA$ the effect of $\mathrm{O}$ on the spectrum is primarily to increase the strength of the TiO lines (see Figures 2 and 3 . The red spectrum therefore offers at the very least a complementary, and arguably a stronger constraint on the $[\mathrm{O} / \mathrm{Fe}]$ abundance.

In the lower right panel we show the results of fitting to the unsmoothed stacked spectra. This is primarily a test of the model in the low $\sigma$ bins, where the unsmoothed spectra have a resolution $\approx 4 \times$ higher than the smoothed spectra. Here again essentially all parameters agree with our standard results to better than 0.03 dex. The largest outlier at low $\sigma$ is $[\mathrm{O} / \mathrm{Fe}]$, but even in this case the disagreement is $<0.1 \mathrm{dex}$.

We have performed an additional test (not shown in the figure), where we masked wavelengths bluer than $4200 \AA$. This was done primarily to test the $[\mathrm{Co} / \mathrm{Fe}]$ result, since there are several strong Co I lines with hyperfine splitting components at $\lambda<4200 \AA$ and many weaker blends at longer wavelengths (see Figure 2). The differences in the derived $[\mathrm{Co} / \mathrm{Fe}]$ ratios between this and our fiducial model is $<0.03$ dex for all $\sigma$ bins except for the lowest bin, where the difference is 0.046 dex. The result for Co seems be insensitive to the particular wavelength range used in the fit.

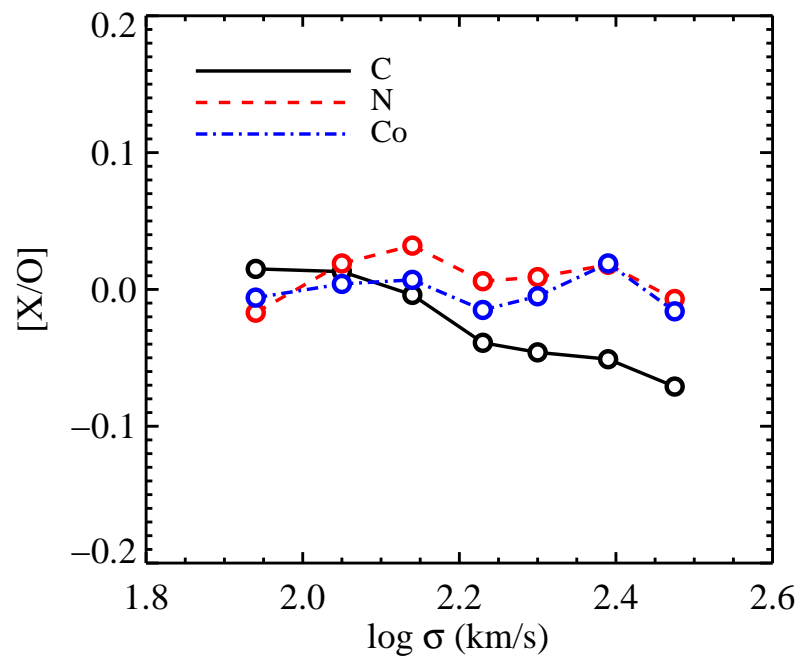

Figure 15. Abundance ratios between $\mathrm{C}, \mathrm{N}, \mathrm{Co}$, and $\mathrm{O}$ as a function of earlytype galaxy velocity dispersion. All of these elements seem to track each other very closely, especially $\mathrm{N}, \mathrm{O}$, and Co. Systematic errors are probably $<0.05$ dex (see Section 5.4).

In summary, the tests performed in this section suggest that the systematic uncertainties in our derived parameters is probably $<0.05$ dex.

\section{COMPARISON TO PREVIOUS WORK}

In this section we provide a comparison to other techniques for deriving stellar population parameters of earlytype galaxies. In Figure 18 we show abundance ratios and light-weighted ages from several groups, including the results presented here, from the EZ_Ages code (Graves \& Schiavon 2008), and from Thomas et al. (2010, T10), Johansson et al. (2012, J12), and Worthey et al. (2013, W13).

The EZ_Ages code fits 7 Lick indices with the SPS models of Schiavon (2007) in order to derive the age, $[\mathrm{Fe} / \mathrm{H}]$, $[\mathrm{C} / \mathrm{Fe}],[\mathrm{N} / \mathrm{Fe}],[\mathrm{Mg} / \mathrm{Fe}]$, and $[\mathrm{Ca} / \mathrm{Fe}]$. The code was run on the exact same stacked spectra analyzed herein, so that any differences can be attributed entirely to differences in modeling techniques. In general the agreement is very good. The $[\mathrm{C} / \mathrm{Fe}]$ and $[\mathrm{N} / \mathrm{Fe}]$ trends are stronger with EZ_Ages compared to the results presented heren. Perhaps the most significant differences are the overall higher $[\mathrm{Mg} / \mathrm{Fe}]$ abundances in EZ_Ages $($ by $\approx 0.07 \mathrm{dex}$ ), and the lower $[\mathrm{Fe} / \mathrm{H}]$ abundances (by $\approx 0.07 \mathrm{dex}$ ).

The results from T10 are based on the SPS models of Thomas et al. (2003b) and Thomas et al. (2004). T10 analyzed a morphologically-selected sample of early-type galaxies from SDSS. They used 25 Lick indices in order to derive three parameters: $[\mathrm{Z} / \mathrm{H}]$, age, and $[\alpha / \mathrm{Fe}]$. We compare their derived $[\alpha / \mathrm{Fe}]$ values to both $[\mathrm{Mg} / \mathrm{Fe}]$ and $[\mathrm{O} / \mathrm{Fe}]$. We find overall satisfactory agreement between their $[\alpha / \mathrm{Fe}]$ values and our $[\mathrm{Mg} / \mathrm{Fe}]$ and $[\mathrm{O} / \mathrm{Fe}]$ values, and also between their ages and ours.

J12 present results from the exact same morphologicallyselected sample of early-type galaxies analyzed in T10, but with the updated SPS models from Thomas et al. (2011). These models allow for variation in the elements $\mathrm{C}, \mathrm{N}, \mathrm{O}$, $\mathrm{Mg}, \mathrm{Ca}$, and $\mathrm{Ti}$, in addition to metallicity and age. They fit their models to the data using 18 Lick indices. The differences between J12 and our own work is the largest of any of the techniques shown in Figure 18 (except for $[\mathrm{O} / \mathrm{Fe}]$ from 


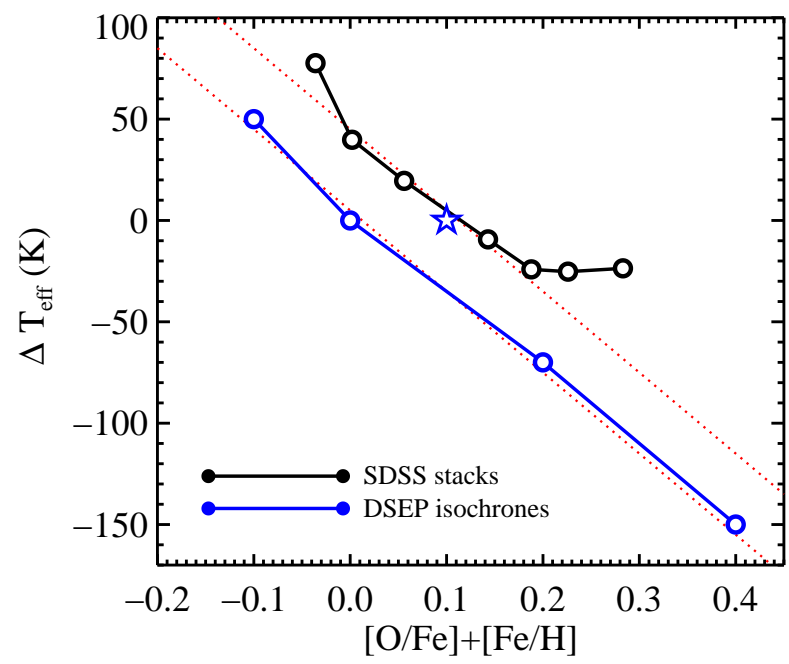

Figure 16. Relation between offset in isochrone $T_{\text {eff }}$ and metal abundance. Results are shown for the stacked SDSS early-type galaxy data and compared to expectations based on theoretical isochrones from the Dartmouth Stellar Evolution Database (DSEP). For the latter, all of the $\alpha$ elements track each other. The theoretical offsets are derived from Figure 6 The star symbol corresponds to the model with $[\mathrm{Fe} / \mathrm{H}]=-0.1$ and $[\alpha / \mathrm{Fe}]=+0.1$ and highlights the fact that $\Delta T_{\text {eff }}$ is not a simple function of $[\mathrm{Z} / \mathrm{H}]$. The dotted lines are offset from each other by $40 \mathrm{~K}$. The excellent agreement in the slope between our modeling and the DSEP predictions is a strong indication that our model is able to reliably measure the distribution of $T_{\text {eff }}$ in early-type galaxies.

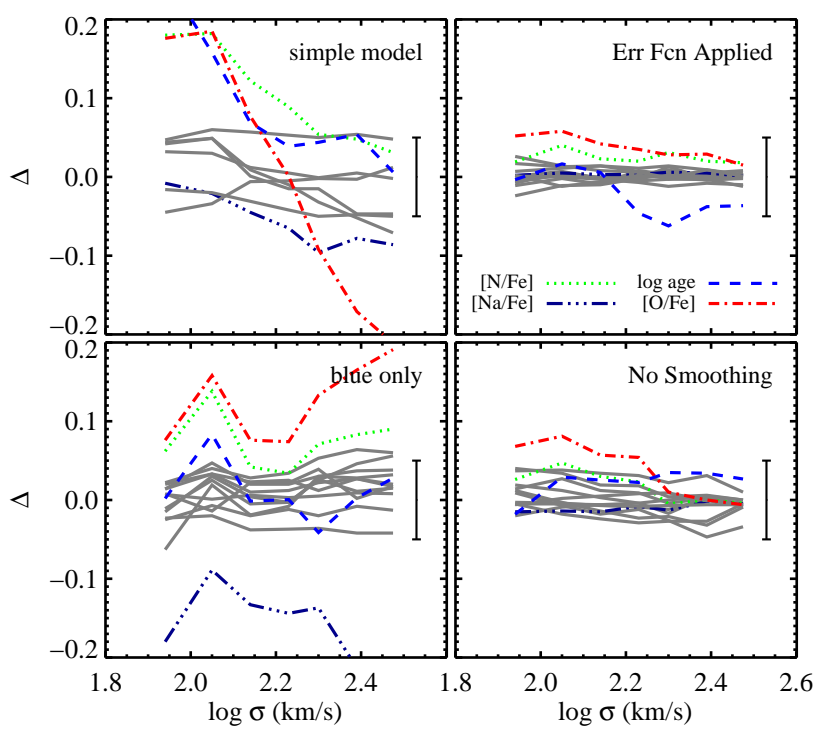

Figure 17. Effect on derived parameters due to varying one or more aspects of our analysis (plotted here as fiducial model minus modified model). All parameters in Table 1 are plotted here (except in the top left panel), but only the most deviant parameters are highlighted. Top Left Panel: Variation between the standard model and a simple model that contains many fewer parameters. Top Right Panel: Variation between the standard model and one in which the mean residuals (averaged over all $\sigma$ bins) are added back into the model. Bottom Left Panel: Variation in parameters when fitting only the blue spectral region $(\lambda<5800 \AA)$. Bottom Right Panel: Variation in parameters when the spectra are not smoothed to a common dispersion of $\sigma=350 \mathrm{~km} \mathrm{~s}^{-1}$. The single error bar in each panel represents an error of \pm 0.05 dex, a value we regard as a plausible upper limit to the systematic errors on the derived parameters.

W13), although the differences rarely exceed 0.1 dex. It is difficult to know if the differences are due to modeling techniques or sample selection, especially at low $\sigma$ where there is likely to be significant scatter between morphological ('earlytype') and emission line ('quiescent') selections.

Very recently, W13 analyzed stacked SDSS early-type galaxy spectra using their SPS models. The stacks are not identical to those analyzed herein, but are very similar and so a direct comparison between $\mathrm{W} 13$ and our results is possible. From Figure 18 it is clear that the ages, $[\mathrm{Mg} / \mathrm{Fe}],[\mathrm{N} / \mathrm{Fe}]$, and $[\mathrm{Ca} / \mathrm{Fe}]$ trends are in good agreement between the techniques, except for the age in the highest $\sigma$ bin in W13. The $[\mathrm{C} / \mathrm{Fe}]$ abundances are also in reasonable agreement. In contrast, the $[\mathrm{O} / \mathrm{Fe}]$ trend derived by $\mathrm{W} 13$ is much steeper than not only our results but also those of $\mathrm{J} 12$ (and T10 if one treats their $[\alpha / \mathrm{Fe}]$ as $[\mathrm{O} / \mathrm{Fe}])$. In addition, their inferred $[\mathrm{Fe} / \mathrm{H}]$ abundances 5 range from -0.05 at low $\sigma$ to -0.7 at high $\sigma$. The results for $[\mathrm{Fe} / \mathrm{H}]$ are so different from the other results in Figure 18 that they were omitted from the Figure for clarity. $\mathrm{W} 13$ fixed $[\mathrm{Ti} / \mathrm{Fe}]=0.0$ throughout their analysis. These authors also presented results for $\mathrm{Si}$, finding $[\mathrm{Si} / \mathrm{Fe}]$ is approximately 0.0 at low $\sigma$ and increases to 0.5 at high $\sigma$. This is also in contrast to our results, in which $[\mathrm{Si} / \mathrm{Fe}]$ increases from 0.0 to 0.16 from low to high $\sigma$. At present we do not know the origins of these discrepancies.

One of the more notable features of Figure 18 is the $[\mathrm{Mg} / \mathrm{Fe}]$ vs. $\sigma$ panel, where there is a $\sim 0.1 \mathrm{dex}$ variation in the normalization of the relation. This variation is significant in the context of using $[\mathrm{Mg} / \mathrm{Fe}]$ to infer a star formation timescale (e.g,. Thomas et al. 2005). Trager et al. (2008) noted that their models produced $[\mathrm{Mg} / \mathrm{Fe}]$ values lower than previous Lick index-based models, and they speculated that this may be due to the different treatment of the response functions. In nearly all previous work on Lick index modeling, three theoretical stellar spectra were combined to estimate the response of the integrated light spectrum to abundance variations (Tripicco \& Bell 1995; Korn et al. 2005). Trager et al., and also Worthey et al. (2013), instead used many stellar spectra covering the isochrone in order to estimate response functions, in a manner similar to what is done in the CvD models. In addition, previous Lick index-based models calculated response functions for an isochrone with an age of 5 Gyr, whereas more recent models use older isochrones (or a range of isochrones appropriate for the ages of the models).

We have estimated the effects of these assumptions by modifying our model to employ only 3 stars (as opposed to the usual 20) to estimate response functions, and also to use stars along a 5 Gyr rather than a $13 \mathrm{Gyr}$ isochrone. The number of stars used to compute response functions has a significant effect. We have created response functions for the $\mathrm{Mg} b$ Lick index due to a factor of two change in $[\alpha / \mathrm{H}]$. A model using 20 stars has a response function in the $\mathrm{Mg} b$ equivalent width (EW) that is $30 \%$ greater than a model that uses only 3 stars. The effect of the age is rather modest, with a change in EW of only $5 \%$, with the 13 Gyr isochrone producing greater EWs. The combination of both effects is a $37 \%$ increase in the response function of the $\mathrm{Mg} b$ index when 20 stars are used along a 13 Gyr isochrone, compared to only 3 stars along a 5 Gyr isochrone. This translates directly into differences in

${ }^{5} \mathrm{~W} 13$ (in addition to T10 and J12) do not constrain [Fe/H] directly but instead estimate $[\mathrm{Z} / \mathrm{H}]$ and the abundance ratios. $[\mathrm{Fe} / \mathrm{H}]$ is thus a derived product and can be roughly estimated via $[\mathrm{Fe} / \mathrm{H}]=[\mathrm{Z} / \mathrm{H}]-A[\alpha / \mathrm{Fe}]$ with $A$ in the range $0.7-0.95$ depending on the detailed abundance pattern (Trager et al. 2000). For the purposes of this discussion we estimated [Fe/H] from W13's data via $[\mathrm{Fe} / \mathrm{H}]=[\mathrm{Z} / \mathrm{H}]-0.9[\mathrm{O} / \mathrm{Fe}]$. 

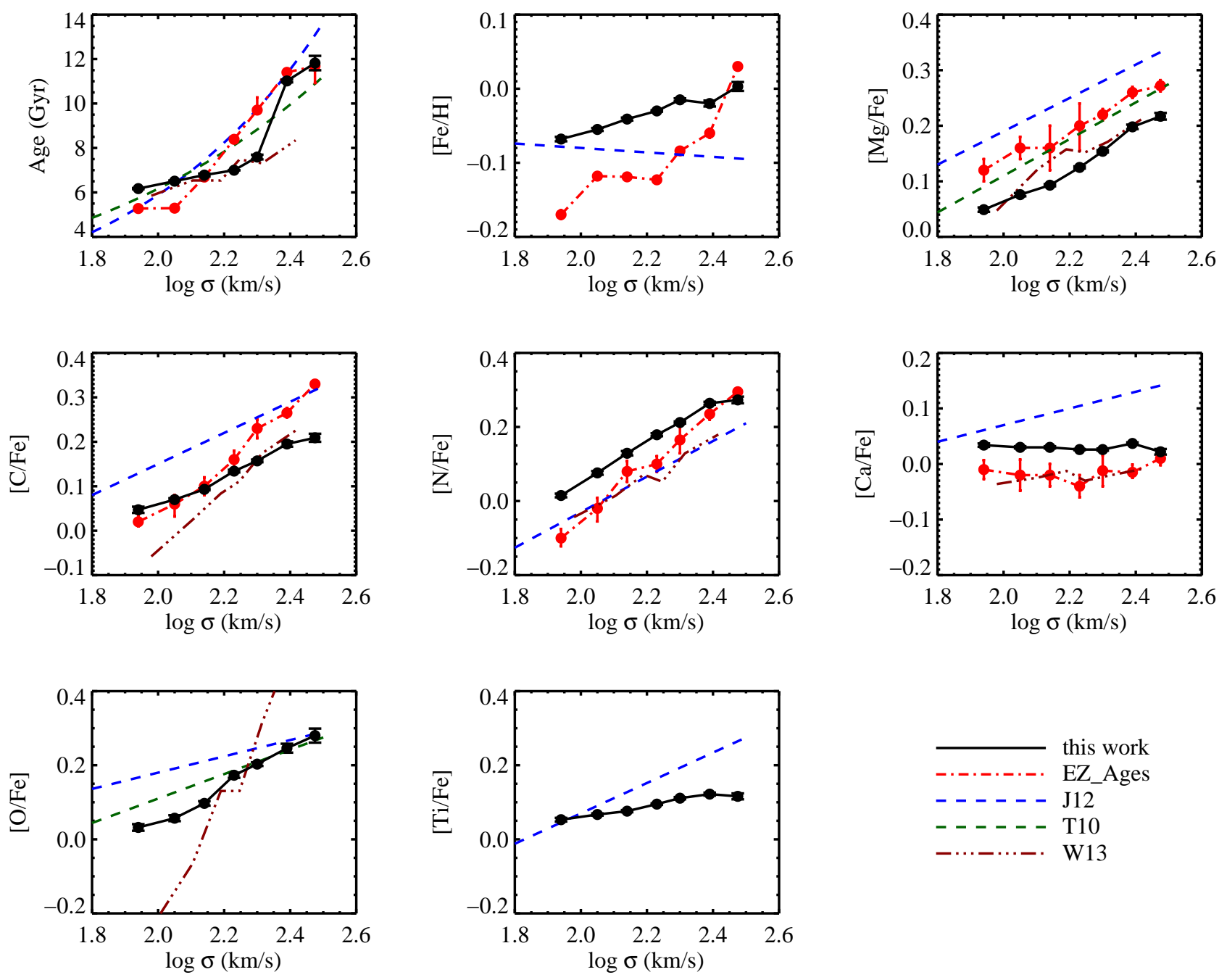

Figure 18. Comparison between different techniques for estimating ages and elemental abundances. Our results are compared to techniques that rely on Lick index fitting, including Graves \& Schiavon (2008, EZ_Ages), Thomas et al. (2010, T10), Johansson et al. (2012, J12), and Worthey et al. (2013, W13). The EZ_Ages results are based on modeling the exact same stacked spectra as used herein, the W13 results are based on similar but not identical stacks, while the $\mathrm{T} 10$ and $\mathrm{J} 12$ results are linear fits to results obtained for $\sim 4000$ SDSS early-type galaxies with light-weighted ages greater than 2.5 Gyr. Note that T10 derive a single $[\alpha / \mathrm{Fe}]$ ratio, which we plot in both the $[\mathrm{Mg} / \mathrm{Fe}]$ and $[\mathrm{O} / \mathrm{Fe}]$ panels, as it is not entirely clear which element their $[\alpha / \mathrm{Fe}]$ parameter is most sensitive to. Qualitatively the derived trends agree well between various methods/groups, although quantitative differences are clearly evident.

the derived $[\mathrm{Mg} / \mathrm{H}]$ abundances, and therefore any model using only a small number of stars along an intermediate-age isochrone will tend to overestimate the $[\mathrm{Mg} / \mathrm{H}]$ abundance.

In summary, there is broad agreement between models for those elements that impart strong, localized changes in low resolution data (especially $\mathrm{C}, \mathrm{N}, \mathrm{Mg}$, and $\mathrm{Ca}$; see Figure 2). In such cases models based on spectral indices seem to perform as well as our model based on full spectrum fitting. The power of fitting the detailed spectrum lies in measuring parameters that either impart a more subtle change in the spectrum (as in the case of $\mathrm{Si}$ and $\mathrm{Mn}$ ), or affect large swaths of the spectrum (as in the case of $\mathrm{O}, \mathrm{Ti}$, and most of the iron peak elements).

\section{DISCUSSION}

The main results from this paper are summarized in Figure 19. where we show the abundance patterns of 13 elements as a function of early-type galaxy velocity dispersion. This is the most comprehensive analysis of the detailed abundance patterns of the stars within distant galaxies to-date. We emphasize that the spectra sample approximately the inner $0.5 R_{e}$, and thus the abundances derived should be representative of the inner regions of the galaxies. In this section we discuss several implications of these results.

The qualitative agreement between our results and those of previous authors is, for the most part, very encouraging. This is significant because our model is a major departure from all previous analyses of the abundance patterns of early-type galaxies. Previous work focused on the analysis of selected spectral indices with theoretical spectral models that were relatively simple with respect to the theoretical spectral libraries used herein. In addition, we have included a large number of additional free parameters in order to marginalize over our ignorance of various aspects of stellar evolution and stellar populations. In spite of these differences in techniques, there is excellent agrement in the trends, including the derived lightweighted ages, the weak variation in $[\mathrm{Fe} / \mathrm{H}]$ and $[\mathrm{Ca} / \mathrm{Fe}]$, and the strong variation in $[\mathrm{Mg} / \mathrm{Fe}],[\mathrm{C} / \mathrm{Fe}]$, and $[\mathrm{N} / \mathrm{Fe}]$. It should not be surprising that there is good agreement between techniques for the elements $\mathrm{C}, \mathrm{N}, \mathrm{Mg}$, and $\mathrm{Ca}$ because these elements impart strong, localized changes in low resolution spec- 


\section{SDSS Early-Type Galaxies}

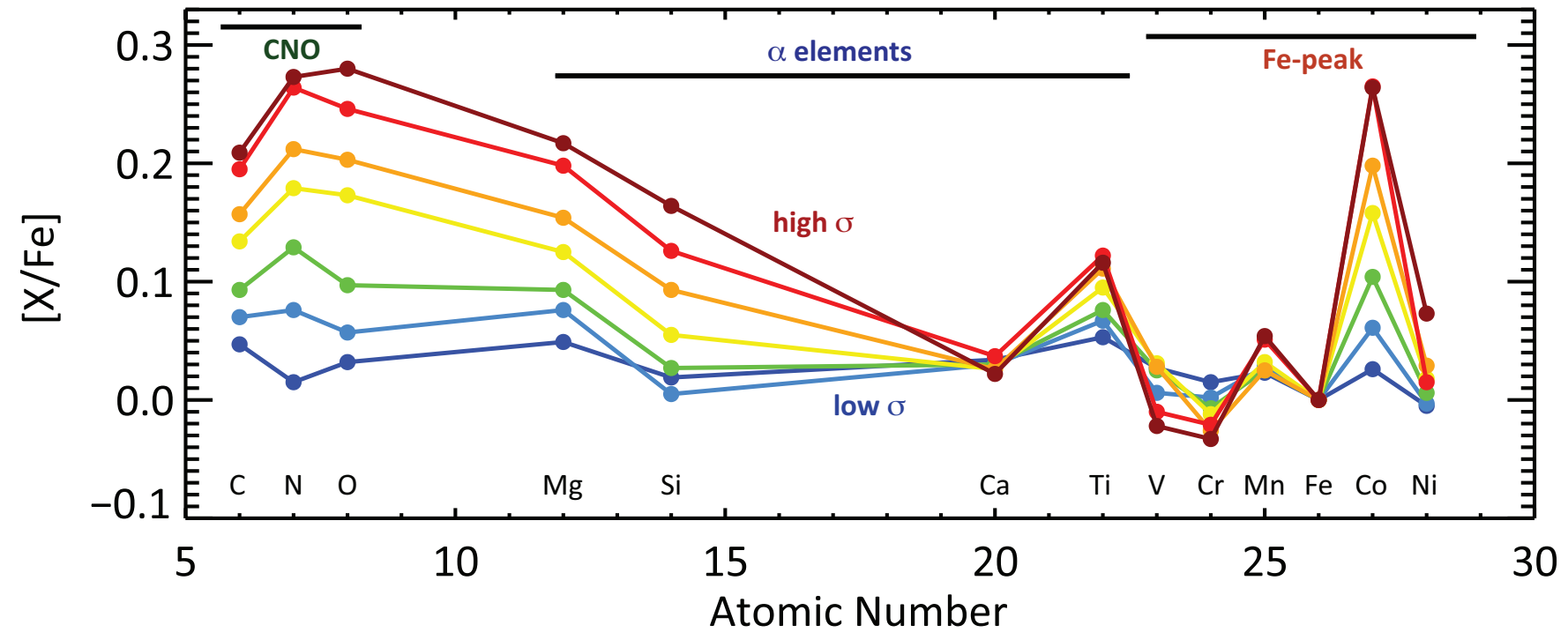

Figure 19. Summary of the abundance trends derived herein from stacked spectra of SDSS early-type galaxies. The sample spans a range in velocity dispersion from $\sigma=90 \mathrm{~km} \mathrm{~s}^{-1}$ to $\sigma=300 \mathrm{~km} \mathrm{~s}^{-1}$. The results for $\mathrm{Na}$ are omitted from this summary diagram, though we note that [Na/Fe] varies from -0.16 to +0.43 from low to high $\sigma$.

tra (see Figure 2). Quantitatively there are differences in the slope and normalization for some of these relations, though the differences rarely exceed $0.1 \mathrm{dex}$ in amplitude. Based on our preliminary investigations, it appears that at least some of the differences can be traced back to the number of theoretical spectra used to estimate response functions to elemental abundance variations (see also Trager et al. 2008). The real power of fitting the detailed spectrum is in the measurement of parameters that either impart a small change in the spectrum (e.g., $\mathrm{Mn}, \mathrm{Sr}, \mathrm{Ba}$ ), or that affect large regions of the spectrum (e.g., O, Ti, V, Cr, Mn, Fe, Co, Ni). For an example of the former, see Conroy et al. (2013).

The standard interpretation of $[\alpha / \mathrm{Fe}]$ is that this ratio is sensitive to the time-scale of star formation (SF), with higher values corresponding to shorter timescales (e.g., Tinsley 1979; Thomas et al. 1999). As mentioned in the Introduction, this ratio is also sensitive to the IMF, the nucleosynthetic yields, the delay time distribution of Type Ia SNe, and the preferential loss of metals via winds. Some chemical evolution models are able to reproduce the observed variation in not only $\mathrm{Mg}$ and $\mathrm{O}$ but also $\mathrm{Si}$ and $\mathrm{Ca}$ based on the assumption that the main variable driving these trends is indeed the SF timescale (Pipino et al. 2009), while others also require variation in other parameters such as the IMF (Arrigoni et al. 2010). In the context of any chemical evolution model, the $[\mathrm{Mg} / \mathrm{Fe}] \mathrm{ra}-$ tio will be correlated with the SF timescale, and so the lower ratios that we derive herein have important implications for the inferred formation timescales of the stars within these massive galaxies. As an example of the effect, Thomas et al. (2005) use the equation $[\alpha / \mathrm{Fe}] \approx \frac{1}{5}-\frac{1}{6} \log \Delta t$, calibrated from the simple chemical evolution model of Thomas et al. (1999), in order to convert their measured $[\alpha / \mathrm{Fe}]$ ratios into constraints on the formation histories of early-type galaxies. In our analysis we find $[\mathrm{Mg} / \mathrm{Fe}]=0.22$ in the most massive galaxies, which, when employing the equation above, corresponds to a timescale of $0.8 \mathrm{Gyr}$. In contrast, the EZ_Ages model and $\mathrm{T} 10$ find $[\mathrm{Mg} / \mathrm{Fe}] \approx 0.27$ at the highest dispersions, corre- sponding to a timescale of 0.4 Gyr. Based on the models of Thomas et al. (2011), J12 find $[\mathrm{Mg} / \mathrm{Fe}] \approx 0.33$ at the highest dispersions, corresponding to a remarkably short timescale of 0.2 Gyr. It is interesting in this context that the $[\mathrm{O} / \mathrm{Fe}] \mathrm{ra}-$ tios derived by $\mathrm{J} 12$ are in much better agreement with our results at the highest dispersions, and so if $[\mathrm{O} / \mathrm{Fe}]$ were used as a tracer of $\alpha$, rather than $\mathrm{Mg}$, then the inferred timescales would be more similar between different groups at high $\sigma$. However, this of course implies that $[\mathrm{O} / \mathrm{Mg}] \neq 0$, and in this case it is not clear that a single parameter " $[\alpha / \mathrm{Fe}]$ " can be used to simply (or reliably) translate derived abundance ratios into SF timescales. Clearly sophisticated chemical evolution models are need to provide further insight.

One of the more interesting trends evident in Figure 19 (see also Figure 15) is the fact that the iron peak element Co closely tracks $\mathrm{O}$ rather than Fe. This is the first measurement of Co in the integrated light spectra of early-type galaxies, and it provides an important constraint on the nucleosynthetic origin of Co. Taken at face value, $[\mathrm{Co} / \mathrm{Fe}] \approx[\mathrm{O} / \mathrm{Fe}]$ implies that Co forms primarily in massive stars, in apparent contrast to the other iron peak elements. In this context it is worth recalling that $\mathrm{Co}$ is also peculiar amongst the iron peak elements in the metal-poor Galactic halo (McWilliam 1997). This may be a coincidence at some level, as the abundance pattern of the very metal-poor halo is probably due exclusively to very metal-poor Type II SNe, in contrast to the processes operating to shape the abundance patterns in massive early-type galaxies. Core-collapse $\mathrm{SNe}$ nucleosynthesis models predict that Co is synthesized by complete Si-burning in the deepest layers, while $\mathrm{Cr}$ and $\mathrm{Mn}$ are produced in the outer incomplete Si-burning layers (Woosley \& Weaver 1995; Nakamura et al. 1999). There is thus a mechanism by which to separate the production of Co from the other iron peak elements. Perhaps more significantly, the Type Ia SNe yields from Nomoto et al. (1984) show a deficit of Co production compared to other iron peak elements. Again, detailed chemical evolution models will be required to sort out these details. 
We reaffirm previous results that the light $\alpha$ elements (e.g., $\mathrm{O}$ and $\mathrm{Mg}$ ) show greater variation than the heavier $\alpha$ elements (e.g., $\mathrm{Ca}$ and Ti). We also find in this work that the variation in $\mathrm{Si}$ is intermediate between the lighter and heavier elements. At least some of these trends can be understood in the context of Type Ia SNe yields. The Nomoto et al. (1984) models produce a large quantity of $\mathrm{Ca}$ (comparable to $\mathrm{Cr}, \mathrm{Mn}, \mathrm{Fe}$, and $\mathrm{Ni}$ ), and much more $\mathrm{Si}$ than either $\mathrm{O}$ or $\mathrm{Mg}$. The modest variation in $\mathrm{Ti}$ is somewhat more difficult to understand in this context, because the Nomoto et al. models produce very little $\mathrm{Ti}$ (comparable to $\mathrm{O}$ and $\mathrm{Mg}$ ). Whether or not the variation in [Ti/Fe] with galaxy dispersion can be reproduced with standard nucleosynthesis yield tables remains to be seen.

Finally we turn to $\mathrm{C}$ and $\mathrm{N}$. As found in previous work, both $[\mathrm{C} / \mathrm{Fe}]$ and $[\mathrm{N} / \mathrm{Fe}]$ increase with increasing dispersion (Schiavon 2007; Graves et al. 2007; Smith et al. 2009; Johansson et al. 2012). We find that both of these elements track $\mathrm{O}$ and $\mathrm{Mg}$ quite closely (in detail $\mathrm{C}$ seems to track $\mathrm{Mg}$ while $\mathrm{N}$ tracks $\mathrm{O}$, but we regard this level of similarity as fortuitous). Johansson et al. (2012) also find that C closely tracks $\mathrm{O}$, with $[\mathrm{C} / \mathrm{O}] \sim 0.0$, but they find sub-solar $[\mathrm{N} / \mathrm{O}]$ values. The fact that $\mathrm{C}$ and $\mathrm{N}$ are tracking the light $\alpha$ elements is probably telling us either that $\mathrm{C}$ and $\mathrm{N}$ form mostly in massive stars, or that there is a lower limit to the star formation timescale such that both massive stars and moderately massive AGB stars (which can produce lots of $\mathrm{C}$ and $\mathrm{N}$ ) are always contributing to the enrichment of the ISM (see also Schiavon 2007; Johansson et al. 2012, who come to similar conclusions). The latter interpretation is consistent with the star formation timescales derived using our $[\mathrm{Mg} / \mathrm{Fe}]$ ratios. Much effort is still needed on the theoretical side to understand the nucleosynthetic origins of these elements in order to use them as cosmological clocks.

\section{SUMMARY}

In this paper we have used the CvD population synthesis model to fit very high quality stacked spectra of SDSS earlytype galaxies spanning a range in velocity dispersion from $90 \mathrm{~km} \mathrm{~s}^{-1}$ to $300 \mathrm{~km} \mathrm{~s}^{-1}$. The novel feature of our approach is fitting the full continuum-normalized optical-NIR spectra ( $4000 \AA-8800 \AA)$, rather than a select number of indices. The model includes variation in age, the abundances of 16 elements, the effective temperature distribution of the stars, the stellar IMF, amongst other parameters. The resulting quality of the fits are very high, with rms residuals ranging from $0.2 \%-0.3 \%$ across the sample. A variety of tests reveal that the systematic uncertainties in our measurements are probably 0.05 dex or less. We now summarize our main results.

- Across the sample from low to high $\sigma$, ages increase from 6 to $12 \mathrm{Gyr},[\mathrm{Fe} / \mathrm{H}]$ increases from -0.07 to 0.00 , and the abundance ratios $[\mathrm{O} / \mathrm{Fe}],[\mathrm{Na} / \mathrm{Fe}],[\mathrm{Mg} / \mathrm{Fe}]$, $[\mathrm{Si} / \mathrm{Fe}],[\mathrm{Ti} / \mathrm{Fe}]$, and $[\mathrm{Co} / \mathrm{Fe}]$ increase from approximately zero to $0.1-0.4$, with $\mathrm{O}, \mathrm{Na}, \mathrm{Mg}$ reaching the highest values. In contrast, $[\mathrm{Ca} / \mathrm{Fe}],[\mathrm{V} / \mathrm{Fe}],[\mathrm{Cr} / \mathrm{Fe}]$, $[\mathrm{Mn} / \mathrm{Fe}]$, and $[\mathrm{Ni} / \mathrm{Fe}]$ remain nearly constant as $\sigma$ increases.

- $\mathrm{N}$ and Co track $\mathrm{O}$ to better than 0.03 dex over the full sample, and $\mathrm{C}$ tracks $\mathrm{O}$ to better than 0.1 dex. Co therefore probably forms predominantly in massive stars. For $\mathrm{N}$ and $\mathrm{C}$ the situation is more complex because these elements probably form in both massive stars and AGB stars. The constant values of $[\mathrm{C} / \mathrm{O}]$ and $[\mathrm{N} / \mathrm{O}]$ therefore either requires $\mathrm{C}$ and $\mathrm{N}$ to form mostly in massive stars or the star formation timescale to never be shorter than several hundred Myr, the lifetime of a $\sim 3 M_{\odot}$ star.

- Our model allows us to measure the shift in the effective temperature of the stars with respect to a solar metallicity isochrone. This shift inversely correlates with the total metallicity, with a slope that is in excellent agreement with theoretical expectations. We are therefore able to measure not only the mean ages and abundance patterns but also the distribution of effective temperatures of the stars in early-type galaxies.

- Comparison of our results to Lick index-based techniques reveals agreement in several quantities, such as age, $[\mathrm{N} / \mathrm{Fe}]$, and $[\mathrm{Ca} / \mathrm{Fe}]$, and less agreement in others including $[\mathrm{C} / \mathrm{Fe}],[\mathrm{Fe} / \mathrm{H}]$, and $[\mathrm{Mg} / \mathrm{Fe}]$. Some of the disagreement may be due to different samples of earlytype galaxies, but the different modeling techniques also seems to play an important role. Perhaps most relevant from the standpoint of broader implications, we find lower $[\mathrm{Mg} / \mathrm{Fe}]$ values at fixed $\sigma$ (by $0.05-0.1$ dex) than some previous work (especially those which employ the Korn et al. 2005, response tables), which we believe is due to our more accurate modeling of spectral variations due to abundance changes. This implies longer formation timescales for massive earlytype galaxies than previously reported.

These results will set the foundation for future studies aimed at constraining the evolutionary histories of galaxies from their detailed abundance patterns. With such a large number of elements precisely measured over a wide range in galaxy mass, it should be possible to place novel constraints not only on the formation histories of these galaxies but also on the nucleosynthetic origins of elements that are difficult to model from first principles.

We thank Bob Kurucz for his continued assistance with the line lists and model atmospheres. We also thank Judy Cohen, Jonas Johansson, Andy McWilliam, Ricardo Schiavon, Scott Trager, Stan Woosley, and Guy Worthey for useful discussions. The referee, Ricardo Schiavon, is thanked for a thorough and very helpful report. This material is based upon work supported by the National Science Foundation under Grant Number 1229745.

Funding for the SDSS and SDSS-II has been provided by the Alfred P. Sloan Foundation, the Participating Institutions, the National Science Foundation, the U.S. Department of Energy, the National Aeronautics and Space Administration, the Japanese Monbukagakusho, the Max Planck Society, and the Higher Education Funding Council for England. The SDSS Web Site is http://www.sdss.org/ The SDSS is managed by the Astrophysical Research Consortium for the Participating Institutions. The Participating Institutions are the American Museum of Natural History, Astrophysical Institute Potsdam, University of Basel, University of Cambridge, Case Western Reserve University, University of Chicago, Drexel University, Fermilab, the Institute for Advanced Study, the Japan Participation Group, Johns Hopkins University, the Joint Institute for Nuclear Astrophysics, the Kavli Institute for Particle Astrophysics and Cosmology, the Korean Scientist Group, the 
Chinese Academy of Sciences (LAMOST), Los Alamos National Laboratory, the Max-Planck-Institute for Astronomy (MPIA), the Max-Planck-Institute for Astrophysics (MPA), New Mexico State University, Ohio State University, University of Pittsburgh, University of Portsmouth, Princeton University, the United States Naval Observatory, and the University of Washington.

\section{REFERENCES}

Abazajian, K. N., Adelman-McCarthy, J. K., Agüeros, M. A., Allam, S. S., Allende Prieto, C., An, D., Anderson, K. S. J., Anderson, S. F., Annis, J., Bahcall, N. A., \& et al. 2009, ApJS, 182, 543

Arrigoni, M., Trager, S. C., Somerville, R. S., \& Gibson, B. K. 2010, MNRAS, 402, 173

Bochanski, J. J., West, A. A., Hawley, S. L., \& Covey, K. R. 2007, AJ, 133, 531

Burstein, D., Faber, S. M., Gaskell, C. M., \& Krumm, N. 1984, ApJ, 287, 586

Carretta, E., Cohen, J. G., Gratton, R. G., \& Behr, B. B. 2001, AJ, 122, 1469

Cenarro, A. J., Gorgas, J., Vazdekis, A., Cardiel, N., \& Peletier, R. F. 2003, MNRAS, 339, L12

Cid Fernandes, R., Mateus, A., Sodré, L., Stasińska, G., \& Gomes, J. M. 2005, MNRAS, 358, 363

Conroy, C. 2013, ARA\&A, 51, 393

Conroy, C. \& van Dokkum, P. 2012a, ApJ, 747, 69

Conroy, C. \& van Dokkum, P. G. 2012b, ApJ, 760, 71

Conroy, C., van Dokkum, P. G., \& Graves, G. J. 2013, ApJ, 763, L25

Cushing, M. C., Rayner, J. T., \& Vacca, W. D. 2005, ApJ, 623, 1115

Dotter, A., Chaboyer, B., Ferguson, J. W., Lee, H.-c., Worthey, G., Jevremović, D., \& Baron, E. 2007, ApJ, 666, 403

Dotter, A., Chaboyer, B., Jevremović, D., Kostov, V., Baron, E., \& Ferguson, J. W. 2008, ApJS, 178, 89

Feltzing, S. \& Johnson, R. A. 2002, A\&A, 385, 67

Graves, G. J., Faber, S. M., \& Schiavon, R. P. 2010, ApJ, 721, 278

Graves, G. J., Faber, S. M., Schiavon, R. P., \& Yan, R. 2007, ApJ, 671, 243

Graves, G. J. \& Schiavon, R. P. 2008, ApJS, 177, 446

Heavens, A. F., Jimenez, R., \& Lahav, O. 2000, MNRAS, 317, 965

Jimenez, R., Bernardi, M., Haiman, Z., Panter, B., \& Heavens, A. F. 2007, ApJ, 669, 947

Johansson, J., Thomas, D., \& Maraston, C. 2012, MNRAS, 421, 1908

Kelson, D. D., Illingworth, G. D., Franx, M., \& van Dokkum, P. G. 2006, ApJ, 653, 159

Korn, A. J., Maraston, C., \& Thomas, D. 2005, A\&A, 438, 685

Kroupa, P. 2001, MNRAS, 322, 231

Kurucz, R. L. 1970, SAO Special Report, 309

-. 1993, SYNTHE spectrum synthesis programs and line data, ed. Kurucz, R. L.

Magic, Z., Serenelli, A., Weiss, A., \& Chaboyer, B. 2010, ApJ, 718, 1378

McWilliam, A. 1997, ARA\&A, 35, 503

Milone, A., Barbuy, B., \& Schiavon, R. P. 2000, AJ, 120, 131

Momany, Y., Ortolani, S., Held, E. V., Barbuy, B., Bica, E., Renzini, A., Bedin, L. R., Rich, R. M., \& Marconi, G. 2003, A\&A, 402, 607

Nakamura, T., Umeda, H., Nomoto, K., Thielemann, F.-K., \& Burrows, A. 1999, ApJ, 517, 193

Nomoto, K., Thielemann, F.-K., \& Yokoi, K. 1984, ApJ, 286, 644

Ocvirk, P., Pichon, C., Lançon, A., \& Thiébaut, E. 2006, MNRAS, 365, 46

Origlia, L., Valenti, E., \& Rich, R. M. 2005, MNRAS, 356, 1276

Peek, J. E. G. \& Graves, G. J. 2010, ApJ, 719, 415
Pipino, A., Chiappini, C., Graves, G., \& Matteucci, F. 2009, MNRAS, 396, 1151

Rayner, J. T., Cushing, M. C., \& Vacca, W. D. 2009, ApJS, 185, 289

Saglia, R. P., Maraston, C., Thomas, D., Bender, R., \& Colless, M. 2002, ApJ, 579, L13

Salaris, M., Chieffi, A., \& Straniero, O. 1993, ApJ, 414, 580

Salpeter, E. E. 1955, ApJ, 121, 161

Sánchez-Blázquez, P., Peletier, R. F., Jiménez-Vicente, J., Cardiel, N., Cenarro, A. J., Falcón-Barroso, J., Gorgas, J., Selam, S., \& Vazdekis, A. 2006, MNRAS, 371, 703

Sbordone, L., Bonifacio, P., Castelli, F., \& Kurucz, R. L. 2004, Memorie della Societa Astronomica Italiana Supplementi, 5, 93

Schiavon, R. P. 2007, ApJS, 171, 146

Schiavon, R. P., Caldwell, N., \& Rose, J. A. 2004, AJ, 127, 1513

Schiavon, R. P., Rose, J. A., Courteau, S., \& MacArthur, L. A. 2005, ApJS, 160, 163

Shetrone, M. D. \& Sandquist, E. L. 2000, AJ, 120, 1913

Smith, R. J., Lucey, J. R., Hudson, M. J., \& Bridges, T. J. 2009, MNRAS, 398,119

Springob, C. M., Magoulas, C., Proctor, R., Colless, M., Jones, D. H., Kobayashi, C., Campbell, L., Lucey, J., \& Mould, J. 2012, MNRAS, 420, 2773

Strauss, M. A. et al. 2002, AJ, 124, 1810

Tautvaišiene, G., Edvardsson, B., Tuominen, I., \& Ilyin, I. 2000, A\&A, 360, 499

Thomas, D., Greggio, L., \& Bender, R. 1999, MNRAS, 302, 537

Thomas, D., Maraston, C., \& Bender, R. 2003a, MNRAS, 343, 279

-. 2003b, MNRAS, 339, 897

Thomas, D., Maraston, C., Bender, R., \& Mendes de Oliveira, C. 2005, ApJ, 621,673

Thomas, D., Maraston, C., \& Johansson, J. 2011, MNRAS, 412, 2183

Thomas, D., Maraston, C., \& Korn, A. 2004, MNRAS, 351, L19

Thomas, D., Maraston, C., Schawinski, K., Sarzi, M., \& Silk, J. 2010, MNRAS, 404, 1775

Tinsley, B. M. 1979, ApJ, 229, 1046

Tojeiro, R., Wilkins, S., Heavens, A. F., Panter, B., \& Jimenez, R. 2009, ApJS, 185, 1

Trager, S. C., Faber, S. M., \& Dressler, A. 2008, MNRAS, 386, 715

Trager, S. C., Faber, S. M., Worthey, G., \& González, J. J. 2000, AJ, 119, 1645

Trager, S. C., Worthey, G., Faber, S. M., Burstein, D., \& Gonzalez, J. J. 1998, ApJS, 116, 1

Tripicco, M. J. \& Bell, R. A. 1995, AJ, 110, 3035

van Dokkum, P. G. \& Conroy, C. 2012, ApJ, 760, 70

VandenBerg, D. A., Bergbusch, P. A., Dotter, A., Ferguson, J. W., Michaud, G., Richer, J., \& Proffitt, C. R. 2012, ApJ, 755, 15

VandenBerg, D. A., Gustafsson, B., Edvardsson, B., Eriksson, K., \& Ferguson, J. 2007, ApJ, 666, L105

Walcher, C. J., Coelho, P., Gallazzi, A., \& Charlot, S. 2009, MNRAS, 398, L44

Woosley, S. E. \& Weaver, T. A. 1995, ApJS, 101, 181

Worthey, G. 1994, ApJS, 95, 107

Worthey, G., Faber, S. M., Gonzalez, J. J., \& Burstein, D. 1994, ApJS, 94, 687

Worthey, G., Tang, B., \& Serven, J. 2013, arXiv:1303.2603

York, D. G. et al. 2000, AJ, 120, 1579

Zoccali, M., Barbuy, B., Hill, V., Ortolani, S., Renzini, A., Bica, E., Momany, Y., Pasquini, L., Minniti, D., \& Rich, R. M. 2004, A\&A, 423, 507 